\title{
Searching for $\mathrm{T}^{\prime}$ in the Little Higgs Model Using Jet Based Information at CMS
}

\author{
T. $\operatorname{Han}^{1}$, S. Kunori ${ }^{2}$,L. Lueking ${ }^{3}$, W. Wu ${ }^{3}$, T. Yetkin ${ }^{4}$ \\ Fermi National Acceleration Laboratory, Batavia, Illinois, U.S.A.
}

January 29, 2006

\begin{abstract}
Recently, the Little Higgs Model has been introduced to try to solve the hierarchy problem. This note describes a study of the Little Higgs Model using jet and $\not_{T}$ based information with the CMS detector at the LHC. The study used Monte Carlo simulations of the physics and CMS detector to explore the capability of CMS to search for $T^{\prime} \rightarrow Z+t$, and $t \rightarrow W+b, W \rightarrow$ two jets with $T^{\prime}$ masses of 1.0 TeV and $1.75 \mathrm{TeV}$. These final state jets have large transverse momentum with very small spatial separation. To deal with such closely spaced hadronic showers, a special jet algorithm was used that was developed to improve the mass resolution and help reduce the background contamination [1]. The focus of this algorithm was to reconstruct parent particles from two hadronic jets in the final state, so the algorithm was modified to work for three jets. A selection criteria was also developed to reduce $\mathrm{Z}$ plus multi-jets background. Using the reconstructed top quark, the transverse mass of the $T^{\prime}$ was formed for $\mathrm{Z} \rightarrow$ neutrinos. The signal significance was calculated for an integrated luminosity of $\left(\sim 300 \mathrm{fb}^{-1}\right)$ and for a $T^{\prime}$ mass of $1.0 \mathrm{TeV}$.
\end{abstract}

\footnotetext{
1) University of Wisconsin, Madison, Wisconsin, USA

2) University of Maryland, College Park, Maryland, USA

3) Fermilab, send comments/questions to weimin@fnal.gov

4) on leave of absence from Cukurova University, Adana, TURKEY.
} 


\section{Introduction}

Current observations in high energy experiments are in excellent agreement with the gauge theory of $S U(3)_{c} \otimes$ $S U(2)_{L} \otimes U(1)_{Y}$ interactions, called the standard model (SM). The many precisely measured electroweak observables favor the existence of a Higgs boson with a mass not much larger than $200 \mathrm{GeV}$. The Higgs boson is a crucial ingredient responsible for the electroweak symmetry breaking and the fermion mass generation in the SM, and thus the next concrete prediction that must be tested at the LHC.

Since there is no symmetry principle in association with the mass of the Higgs boson in the SM, quantum (loop) corrections tend to drag the mass to a higher energy scale, possibly to the Planck scale. This is the so-called hierarchy problem. In order to stabilized the well-established electroweak scale against the severe radiative corrections, one needs new dynamics to set in not far above the electroweak scale. This is the major motivation for physics beyond the standard model. Super-symmetric theories introduce a new space-time symmetry and quadratically divergent quantum corrections are canceled among bosonic and fermionic partners to stabilize the electroweak scale. The "Little Higgs" theories [2, 3], as an alternative, introduce new heavy fermions and gauge bosons to cancel the quadratic divergence at least at the 1-loop level. In general, if we take the SM as a low energy effective theory with a naturally light Higgs boson, new states are needed to cure the ill-behaved quantum corrections.

The most severe quantum corrections to the Higgs mass is due to the top-quark loop, because of the large Higgstop Yukawa coupling $\lambda_{t} \sim 1$. A new state with related coupling to $\lambda_{t}$ must show up below about $3 \mathrm{TeV}$ to keep the Higgs mass naturally light. In the little Higgs scenarios, it is the vector-like heavy state $T$, and this is robust prediction for the little Higgs theory. We thus set out to explore its observability at the LHC experiments.

There are many proposed channels for the "Little Higgs Model" study[4], the motivation of choice to pick this channel $T^{\prime} \rightarrow Z+t$ and $t \rightarrow W+b$, then $W \rightarrow$ jet + jet as our study of the "Little Higgs Model", is

a) Fermilab is responsible for the Hadronic Calorimeter(HCAL) of CMS, We want to know how well of the capabilities of the HCAL to measure the very highly boosted jets in $\mathrm{TeV}$ mass region.

b) We have developed the new "cluster-mass" algorithm[1] for the two jets case, we want to extend this algorithm to the three jets case as final state using same principal, so the new algorithm will provide good opportunity to study multi-jets topics. Our focus is on the jets, so we choose $\mathrm{Z}$ decay into neutrinos, not decay to the jets in order to simplify the study.

In this case, we will be dealing with the $\mathbb{E}_{T}$, which will use the information of current jet/met root file provided, which was simply calculated based on the calorimeter towers[5].

\section{Kinematics and Cross section of $T^{\prime} \rightarrow Z+t$ in the Little Higgs Model}

For the Little Higgs Model, the production and decay $T^{\prime} \rightarrow Z+t$ was simulated using the Monte Carlo generator Pythia 6.227 in p-p collisions at the LHC CM energy of $14.0 \mathrm{TeV}$. The $\mathrm{Z}$ boson was decayed into e+e- or neutrinos, and the top decayed into a $\mathrm{W}$ boson and $\mathrm{b}$ quark. The $\mathrm{W}$ boson was forced to decay into two quarks. These events were then processed using a current CMS software simulation of the detector: CMKIN 4.3.1, OSCAR 3.6.5, and ORCA 8.7.3. The associated JetMet ntuple was then produced with Jet/Met Rootmaker[5].

The cross section for this process depends on both the mass of the $T^{\prime}$ and the $\mathrm{t}-\mathrm{T}$ mixing, and thus on the ratio of the parameters $\lambda 1 / \lambda 2$ in the Little Higgs Model. According to the calculation in [4], for T' mass at $1.0 \mathrm{TeV}$, the production cross section is between $48 \mathrm{fb}$ and $770 \mathrm{fb}$, for a $\lambda 1 / \lambda 2$ ratio between 0.5 and 2 . Specifically, for $\lambda 1 / \lambda 2=1$, the production cross section is $192 \mathrm{fb}$, and for $\lambda 1 / \lambda 2=2,770 \mathrm{fb}$. For a $T^{\prime}$ mass of $1.75 \mathrm{TeV}$, the production cross section is correspondingly $27 \mathrm{fb}$ and $102 \mathrm{fb}$ respectively, about factor of seven smaller than for a $T^{\prime}$ mass of $1.0 \mathrm{TeV}$. Furthermore, there are three possible decays of the $T^{\prime}: T^{\prime} \rightarrow Z+t, T^{\prime} \rightarrow W+b$, 


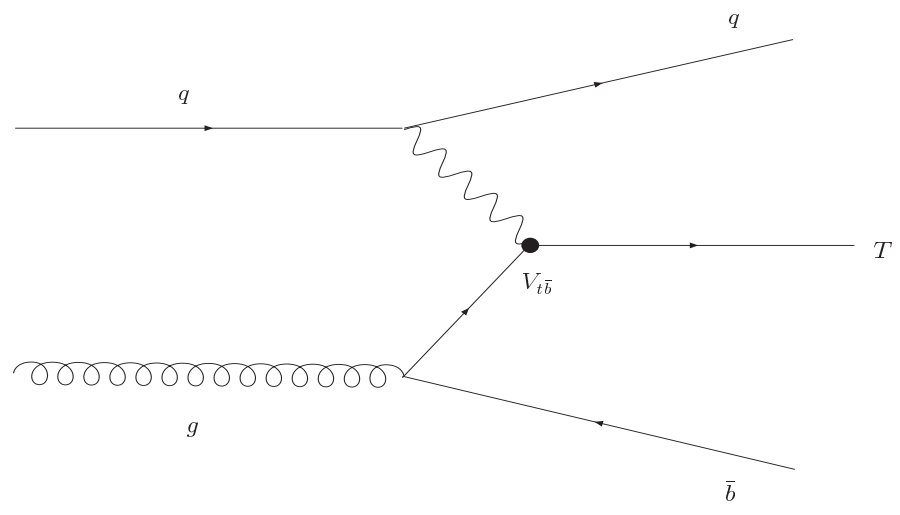

Figure 1: Main diagram for $T^{\prime}$ production

$T^{\prime} \rightarrow$ top + Higgs, and only $1 / 4$ of the decays are $\mathrm{Z}+\mathrm{t}$. Since the hadronic breaching ratio of the $\mathrm{W}$ boson is $2 / 3$, and the branching ratio for the $\mathrm{Z}$ boson into neutrinos is about $20 \%$ (electrons, $3.3 \%$ ), the cross section times branching ratio is only $25.6 \mathrm{fb}(4.3 \mathrm{fb})$. Therefore, the final results are presented for a $T^{\prime}$ mass of $1.0 \mathrm{TeV}$ and $\lambda 1 / \lambda 2=2$, which requires significantly less LHC integrated luminosity.

The cross section provided by Pythia, is very close to the number calculated by [4] ( 150 vs 192 for $\lambda 1 / \lambda 2=1$ ). In this note, the cross section in [4] was used for the final normalization. Figure 1 shows the main diagram for single $\mathrm{T}$ ' production via $\mathrm{W}$-emission and gluon splitting in the $\mathrm{t}$-channel. Along with the $\mathrm{T}$ ', the process produces two forward jets. These forward jets will be used to reduce the backgrounds.

Figures 2 and 3 show that the $T^{\prime}$ is produced with larger momentum $(\mathrm{P})$, large longitudinal momentum $(\mathrm{Pz})$ and small transverse momentum $\left(P_{T}\right)$, as expected. Figures 4 and 5 show that the $P_{T}$ of the $\mathrm{Z}$ boson is peaked at around the half of the $T^{\prime}$ mass. Figures 6 and 7 show that the $P_{T}$ of $\mathrm{W}$ boson from the $\mathrm{t}$ decay is quite large, so the decay products have large $P_{T}$ also, as shown in Figures 8 and 9 . Figures 10 and 11 show that the $\mathrm{b}$ quark from the t decay is produced mainly in central region. However, the $P_{T}$ is also very large, which makes sufficient b-tagging of these $\mathrm{b}$ jets difficult. Using the current $\mathrm{b}$-tagging algorithms, tagging such high $P_{T} \mathrm{~b}$ jets is almost impossible with any reasonable rate.

Figures 12 and 13 show the distance in $\eta$ - $\phi$ space measured as $\left.\delta R=\sqrt{(} \delta \eta^{2}+\delta \phi^{2}\right)$, between u quark and d quark, $\mathrm{u}$ quark and $\mathrm{b}$ quark and $\mathrm{b}$ quark and $\mathrm{d}$ quark (where the $\mathrm{u}$ and $\mathrm{d}$ refer to the two quarks of $\mathrm{W}$ boson decay). The distances are mostly smaller than 1 (the minimum of $\Delta R$ of these values is shown in the top-left plot). Regular cone algorithm with cone size of 0.5 can not separate these jets from the top decay. A smaller cone size will lose jet energy significantly, and worsen the mass resolution. Clearly, the regular cone algorithms will do a poor job reconstructing these large $P_{T}$, closely spaced jets. Figures 14 and 15 show that the $\Delta R$ between the $\mathrm{W}$ and $\mathrm{b}$ from the $t$ decay, is also mostly smaller than 1 , so the products of $t$ quark decay are basically merged together as one "fat" jet.

As shown in Figures 16 and 17, with a cone size of 0.5, the number of towers in the overlapping region for these three jets averages about 26 for a $T^{\prime}$ mass of $1.0 \mathrm{TeV}$, and 52 for a $T^{\prime}$ mass of $1.75 \mathrm{TeV}$. The HCAL's tower size is 0.087 , so that the total number of towers for one jet within a cone size of 0.5 is about 100 . Clearly, a conventional cone algorithm will not measure the jet energies correctly with such a large number of overlapped towers. This is a serious challenge to any algorithm and is a main focus of this study.

Figures 18 and 19 show the missing transverse momentum for the case where the $\mathrm{Z}$ boson decays to neutrinos. As expected, the $\not_{T}$ is peaked at around the half of the $T^{\prime}$ mass. 

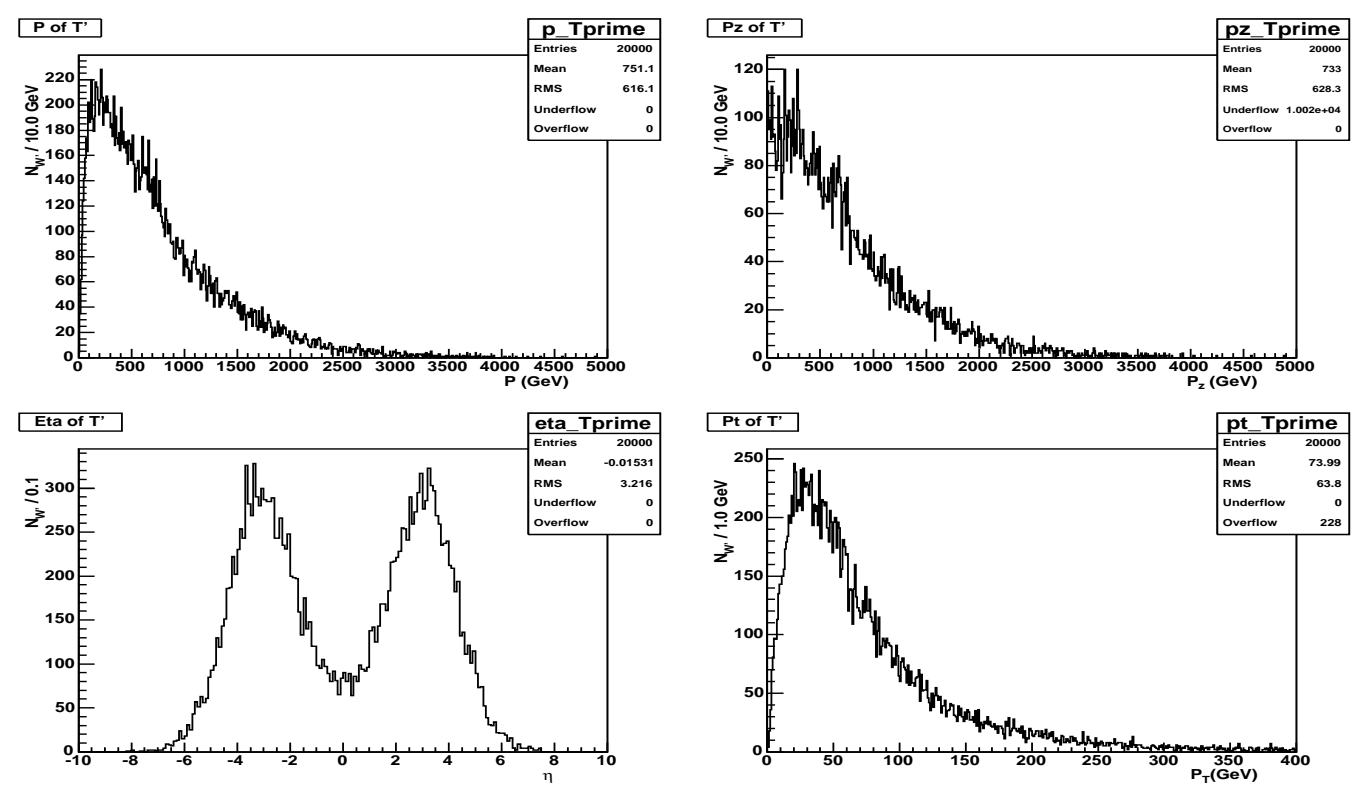

Figure 2: $T^{\prime}$ kinematics for $1 \mathrm{TeV}$ mass
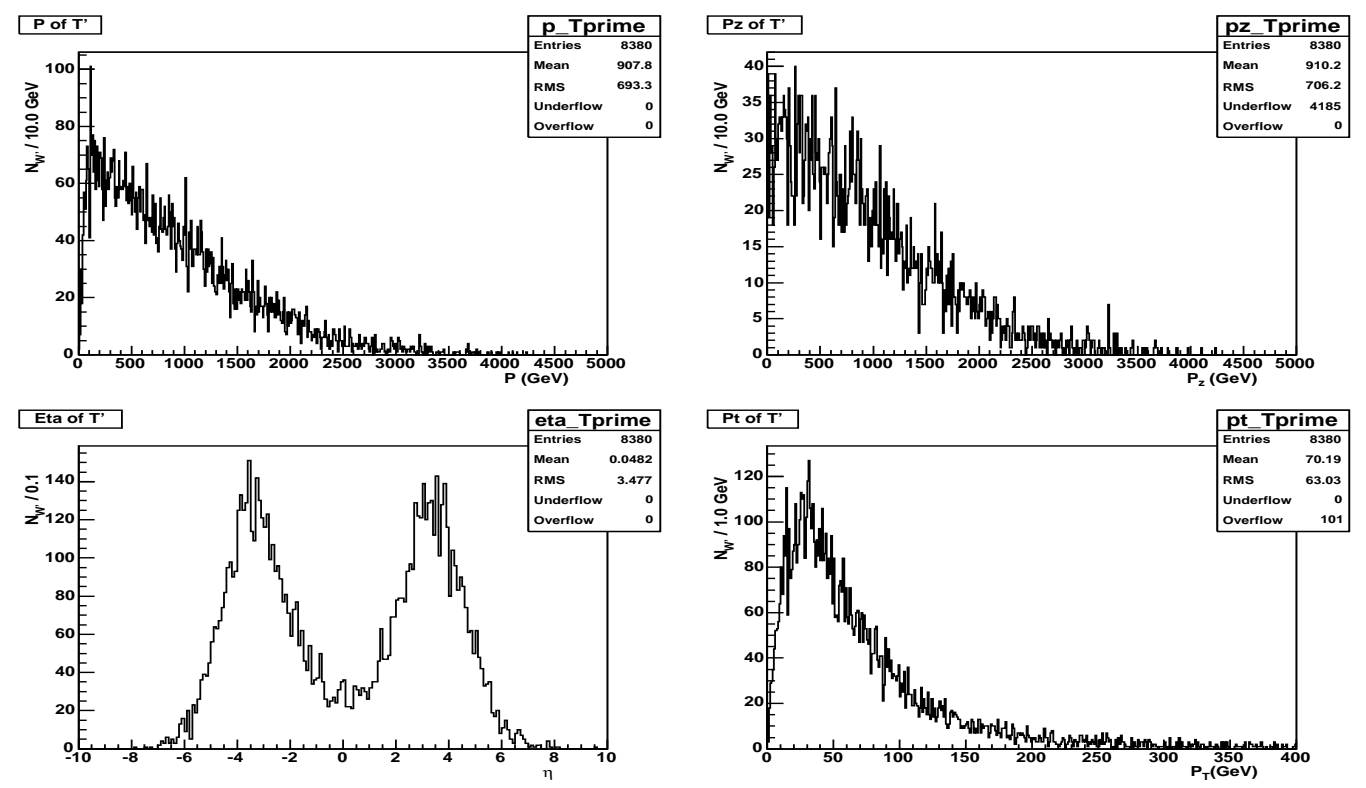

Figure 3: $T^{\prime}$ kinematics for $1.75 \mathrm{TeV}$ mass 


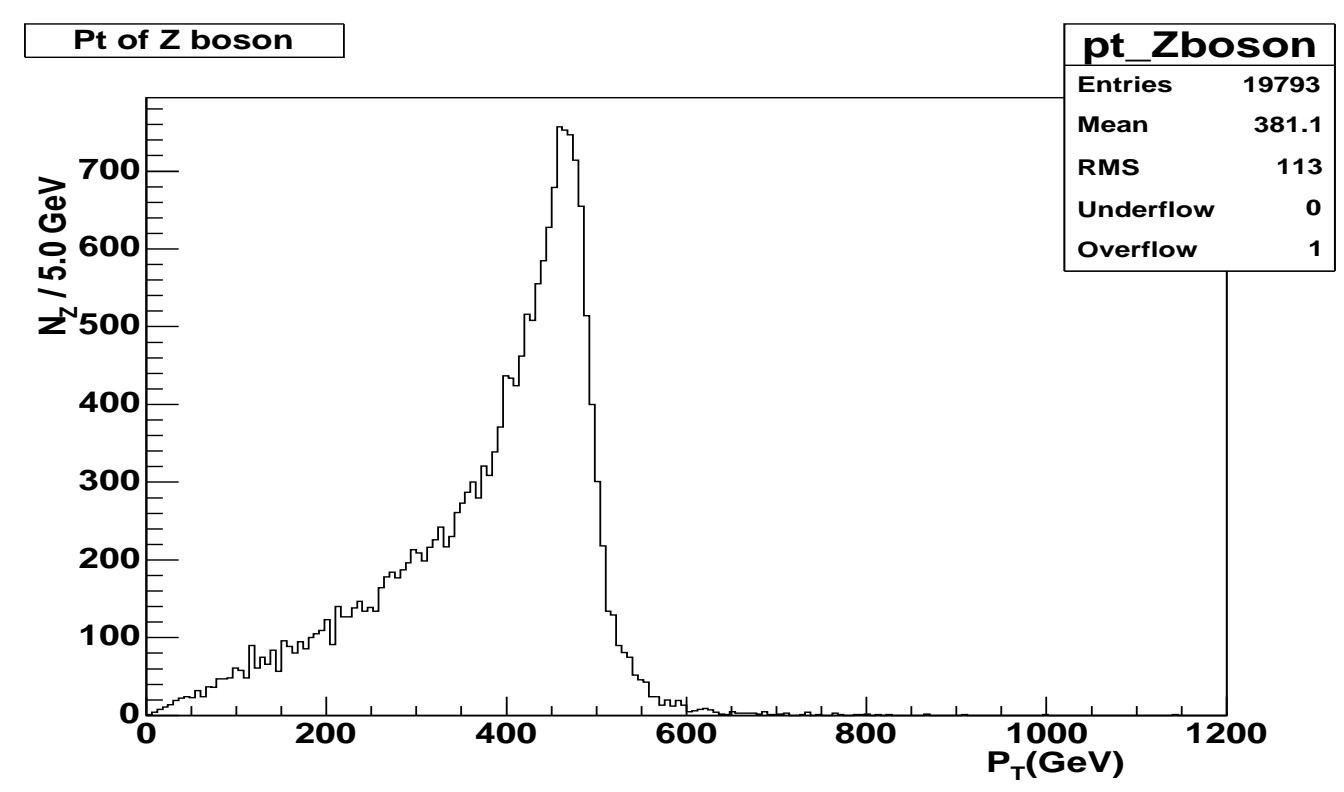

Figure 4: $\mathrm{Z}$ boson property for $T^{\prime}$ mass of $1 \mathrm{TeV}$

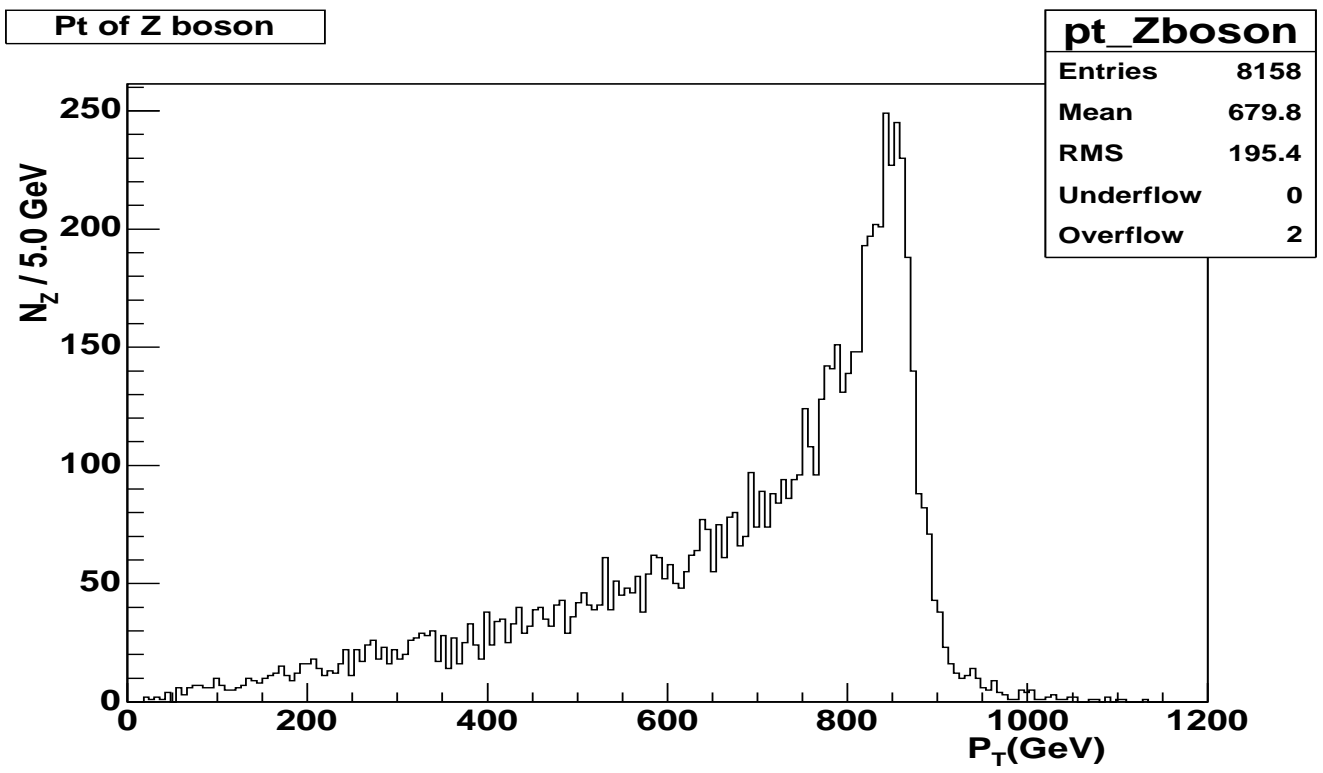

Figure 5: $\mathrm{Z}$ boson property for $T^{\prime}$ mass of $1.75 \mathrm{TeV}$ 


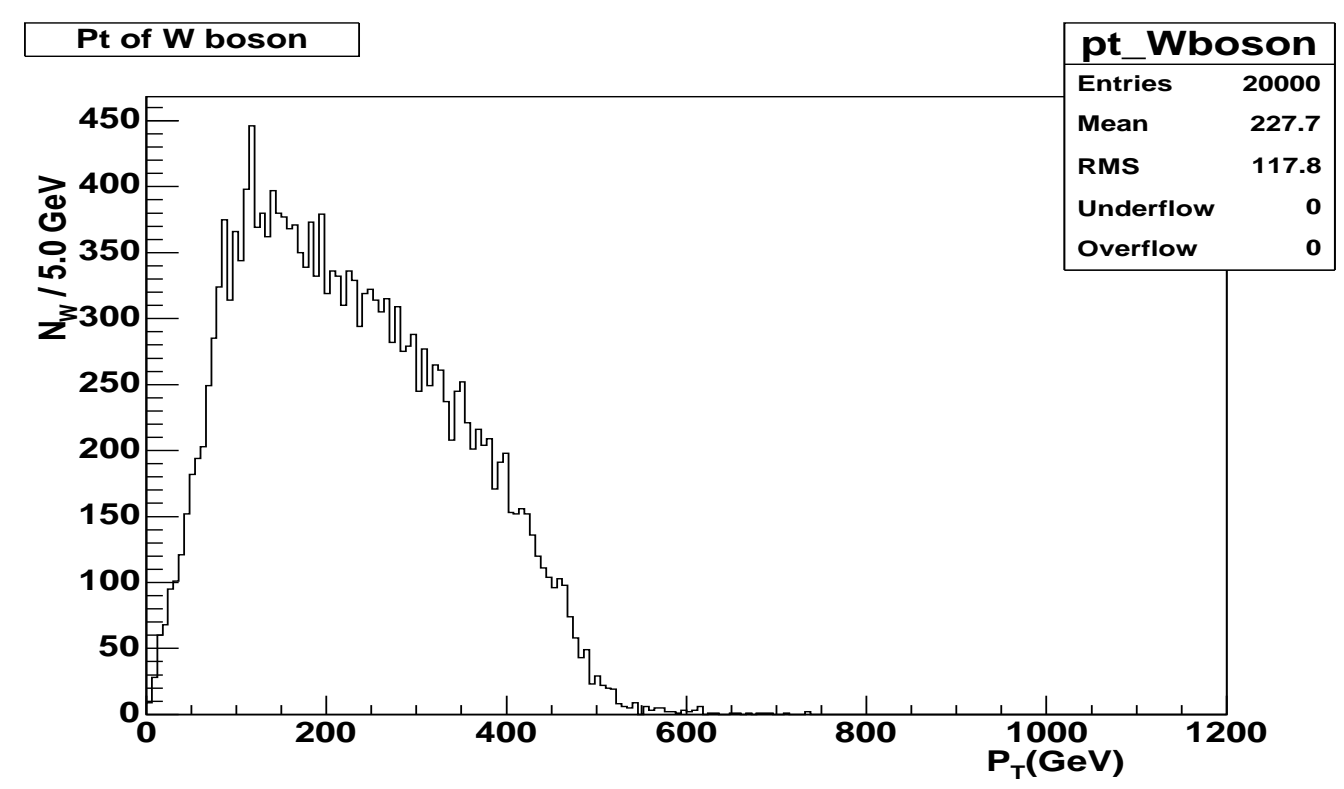

Figure 6: $\mathrm{W}$ boson property for $T^{\prime}$ mass $1 \mathrm{TeV}$

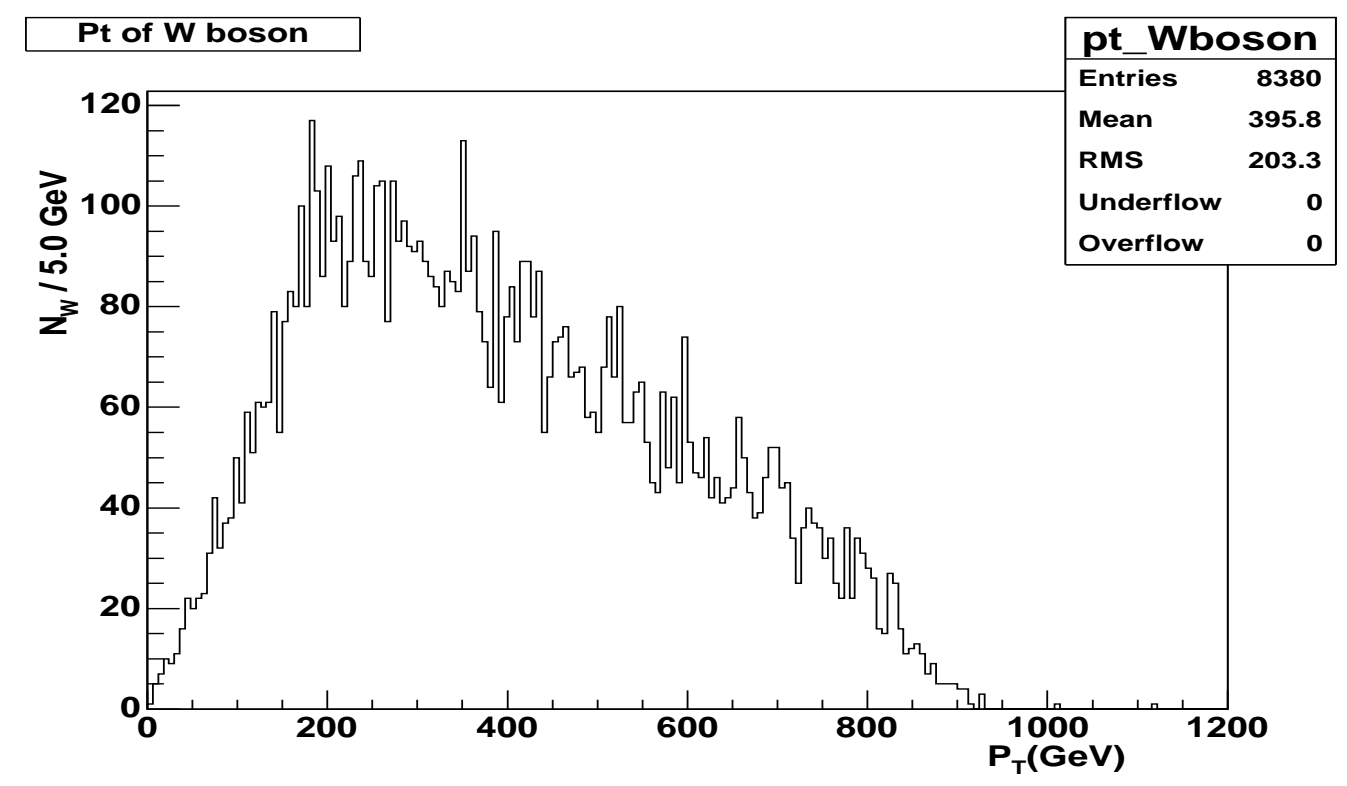

Figure 7: W boson property for $T^{\prime}$ mass $1.75 \mathrm{TeV}$ 


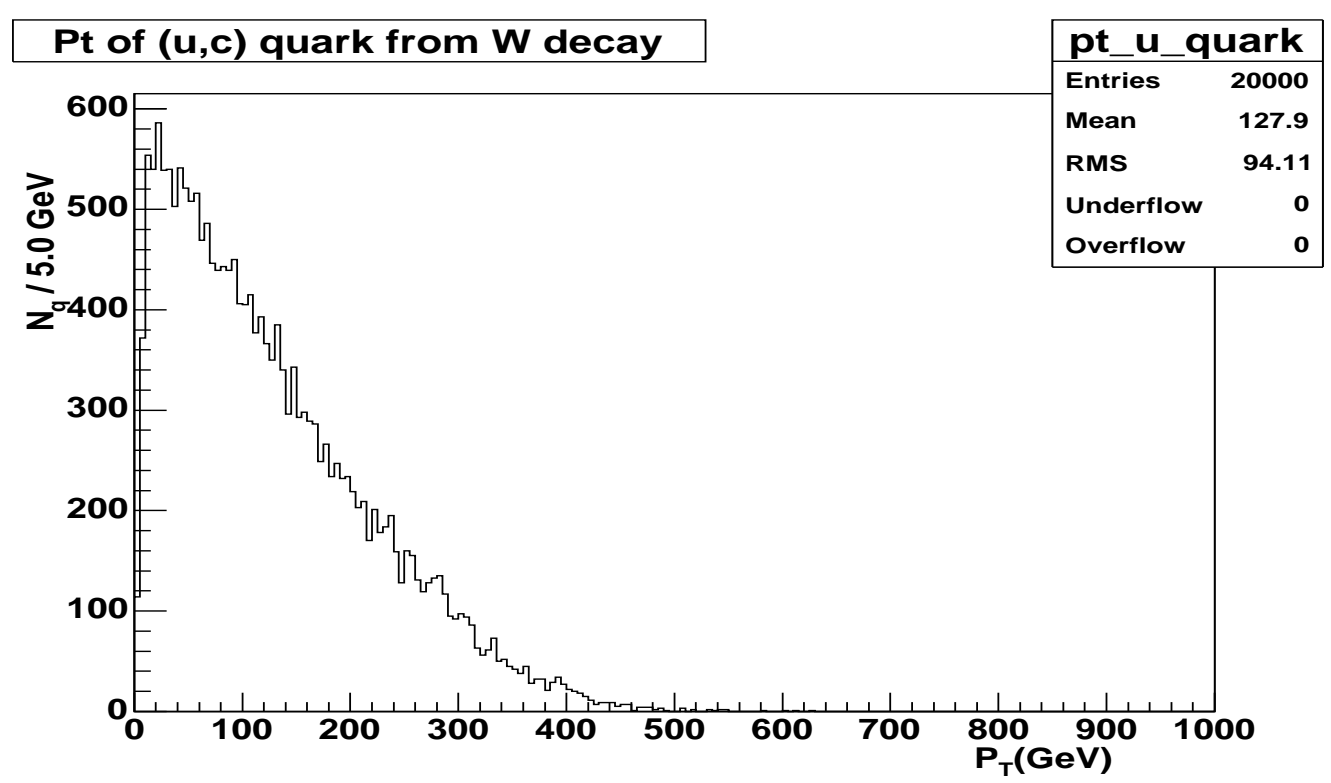

Figure 8: $P_{T}$ of quarks from $\mathrm{W}$ decay for $T^{\prime}$ mass $1 \mathrm{TeV}$

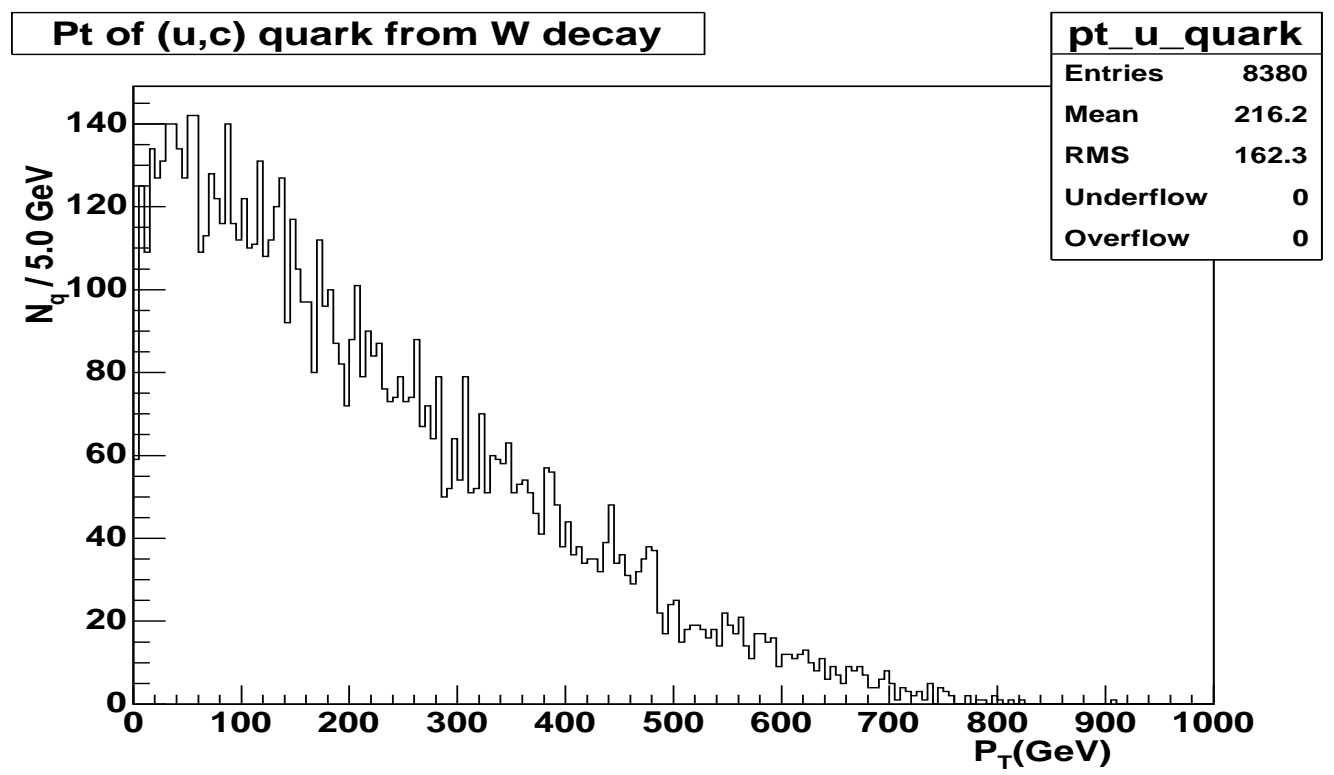

Figure 9: $P_{T}$ of quarks from $\mathrm{W}$ decay for $T^{\prime}$ mass $1.75 \mathrm{TeV}$ 


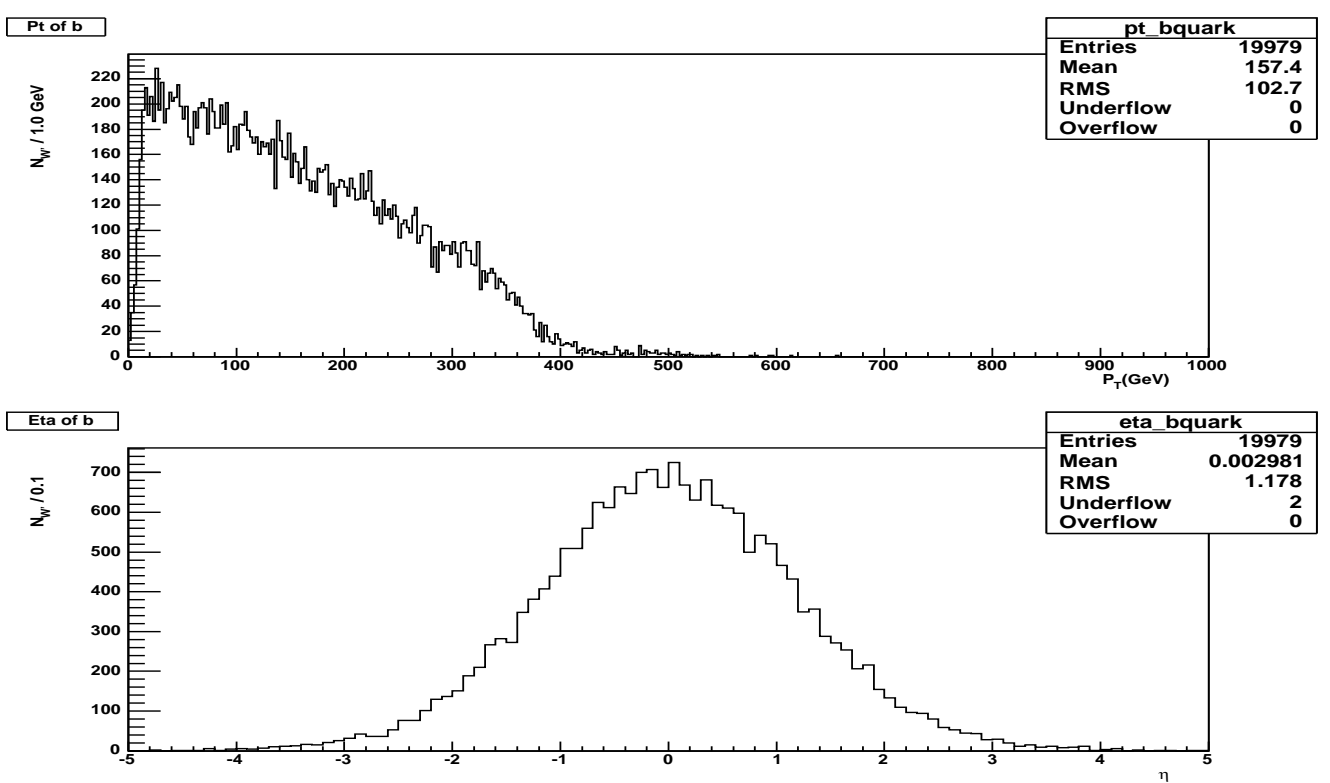

Figure 10: $P_{T}$ of b quark from $\mathrm{t}$ decay for $T^{\prime}$ mass $1 \mathrm{TeV}$

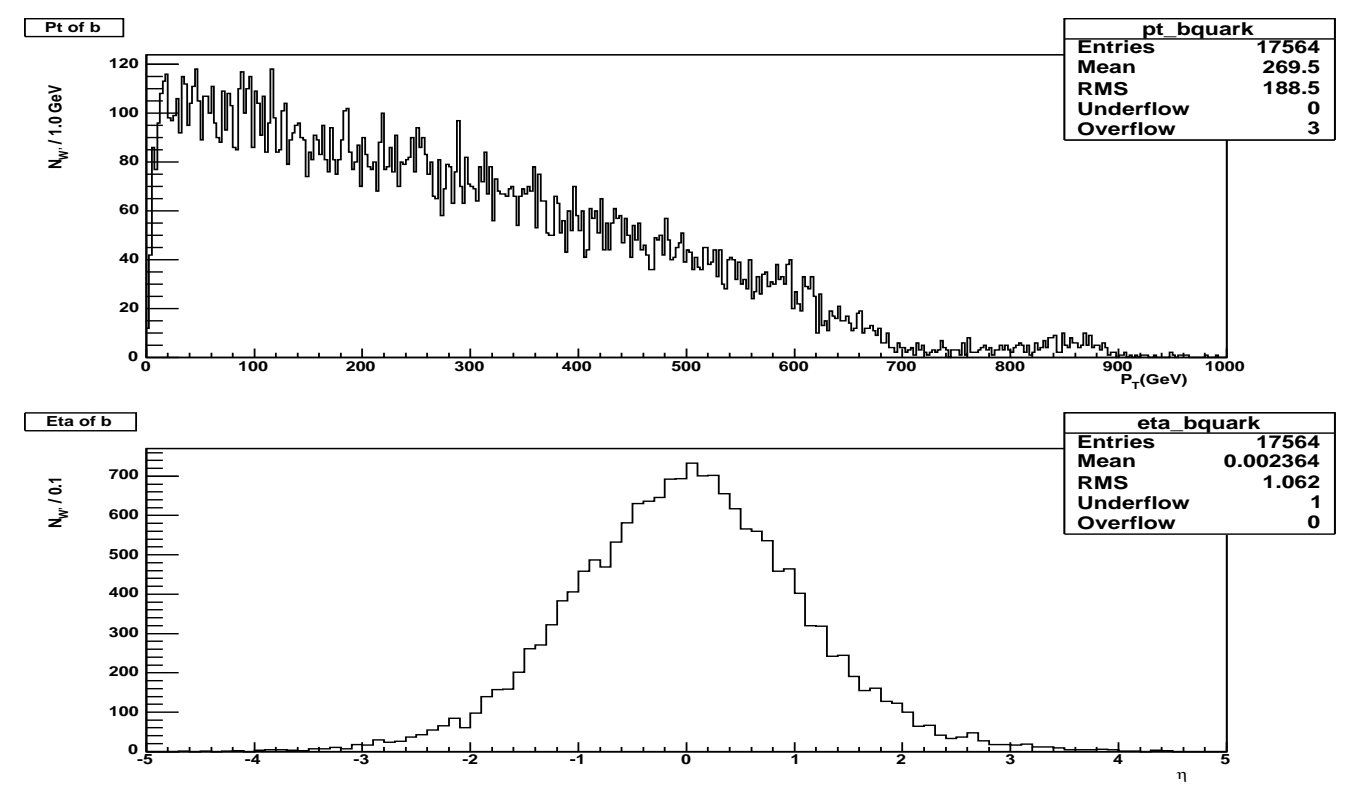

Figure 11: $P_{T}$ of b quarks from $\mathrm{t}$ decay for $T^{\prime}$ mass $1.75 \mathrm{TeV}$ 

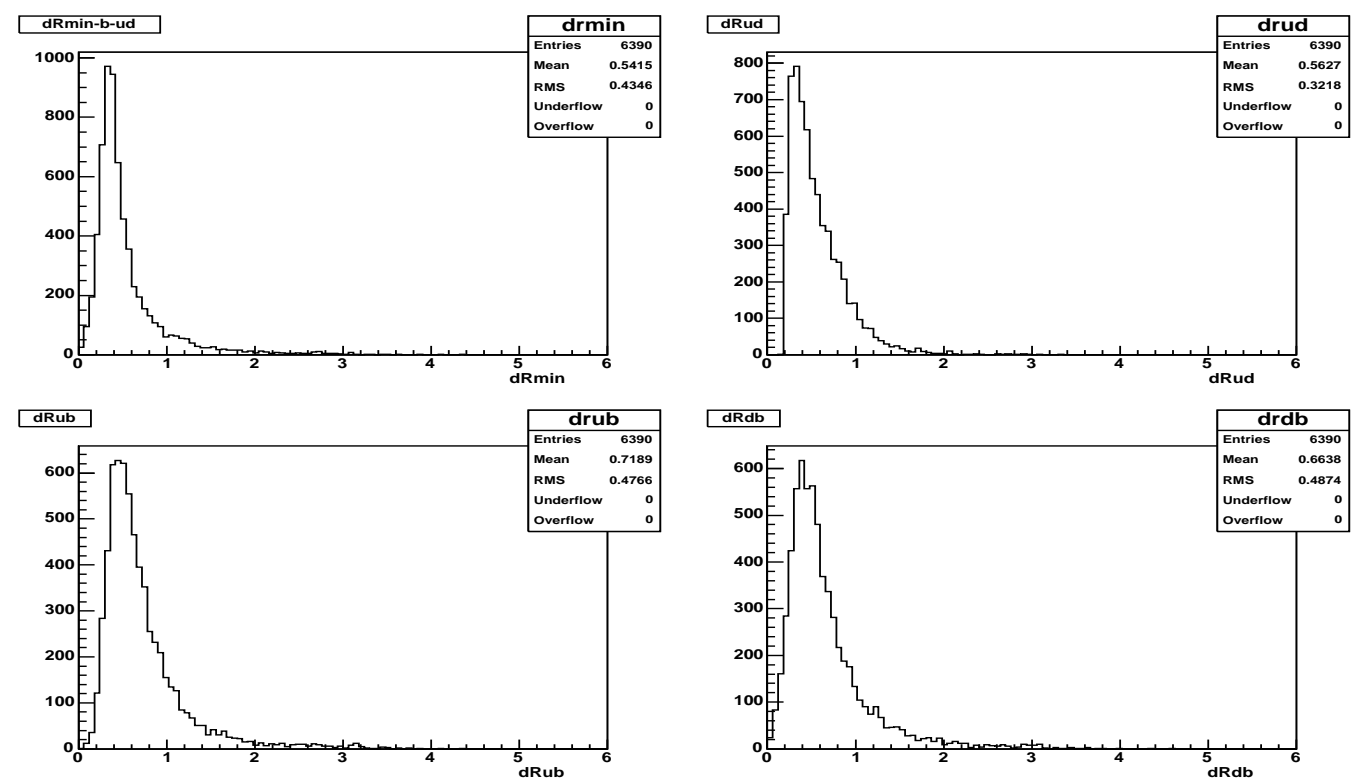

Figure 12: $\Delta R$ in $\eta$ - $\phi$ space for $1 \mathrm{TeV} T^{\prime}$
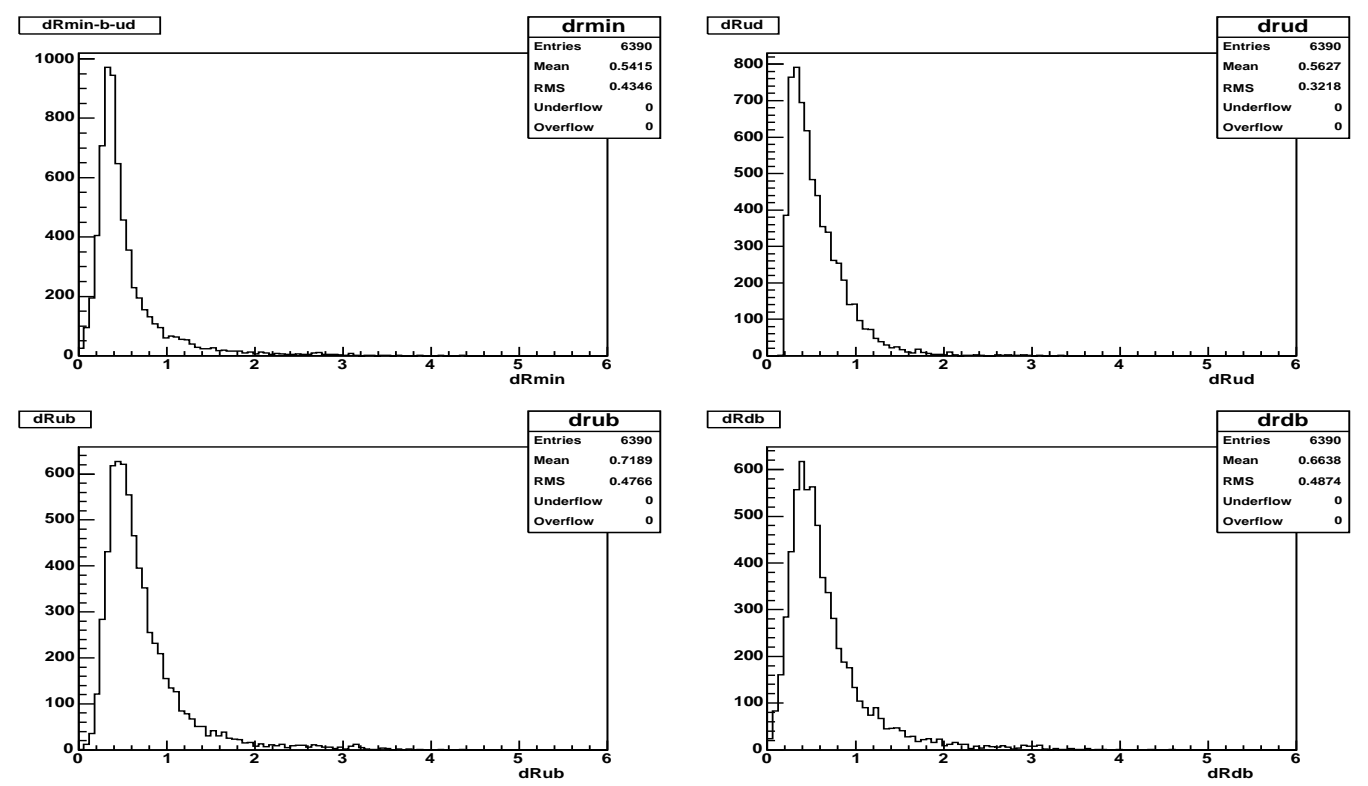

Figure 13: $\Delta R \eta-\phi$ space for $1.75 \mathrm{TeV} T^{\prime}$ 


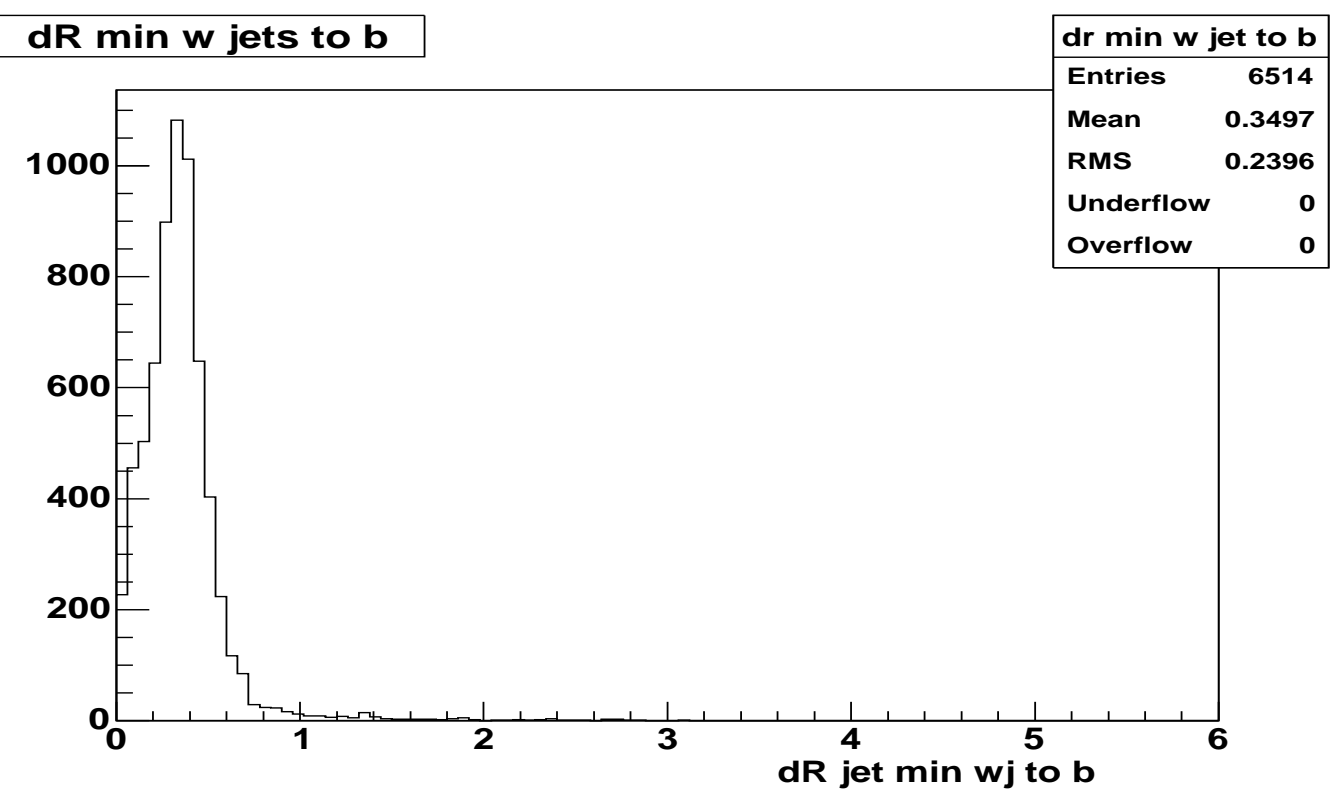

Figure 14: $\Delta R$ between b quark and the $\mathrm{W}$ for $T^{\prime}$ mass $1 \mathrm{TeV}$

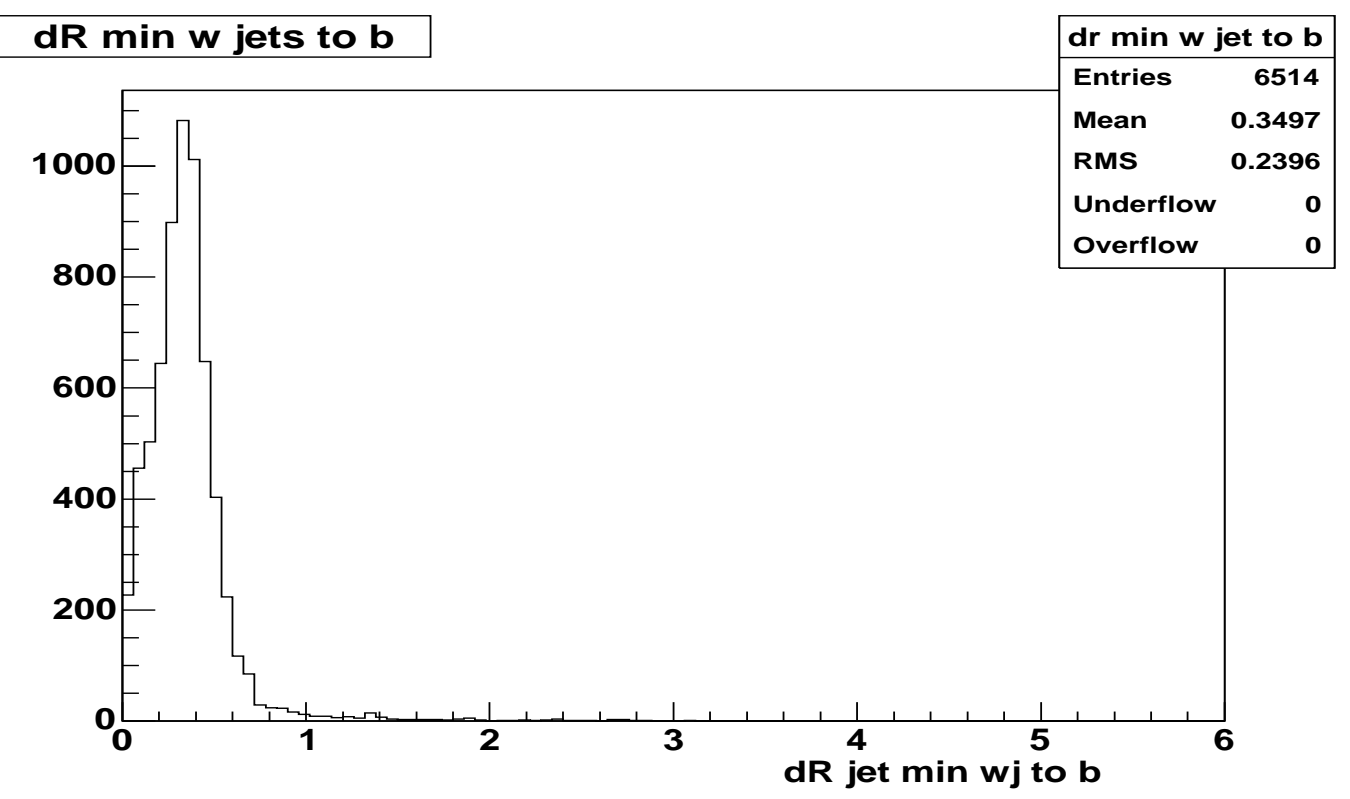

Figure 15: $\Delta R$ between b quark and the $\mathrm{W}$ for $T^{\prime}$ mass $1.75 \mathrm{TeV}$ 


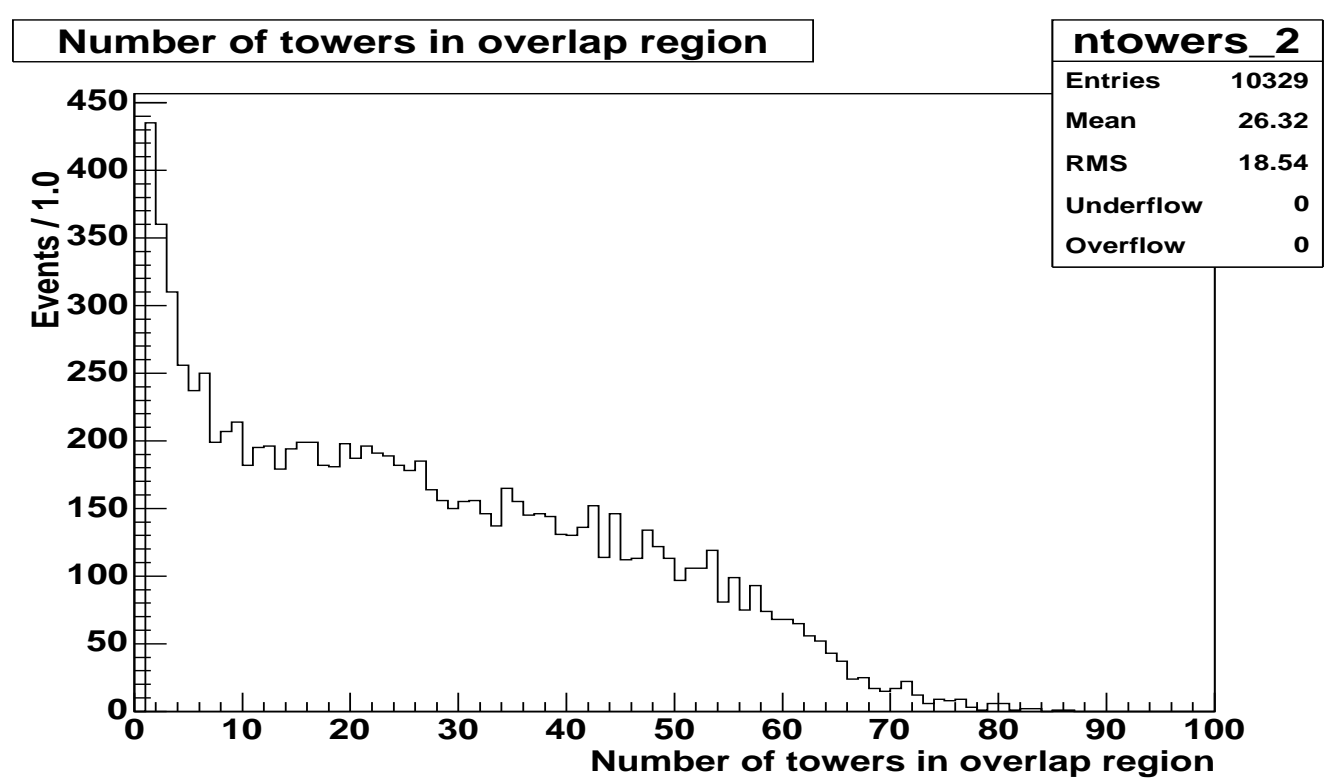

Figure 16: Number of towers overlapped for $T^{\prime}$ mass $1 \mathrm{TeV}$

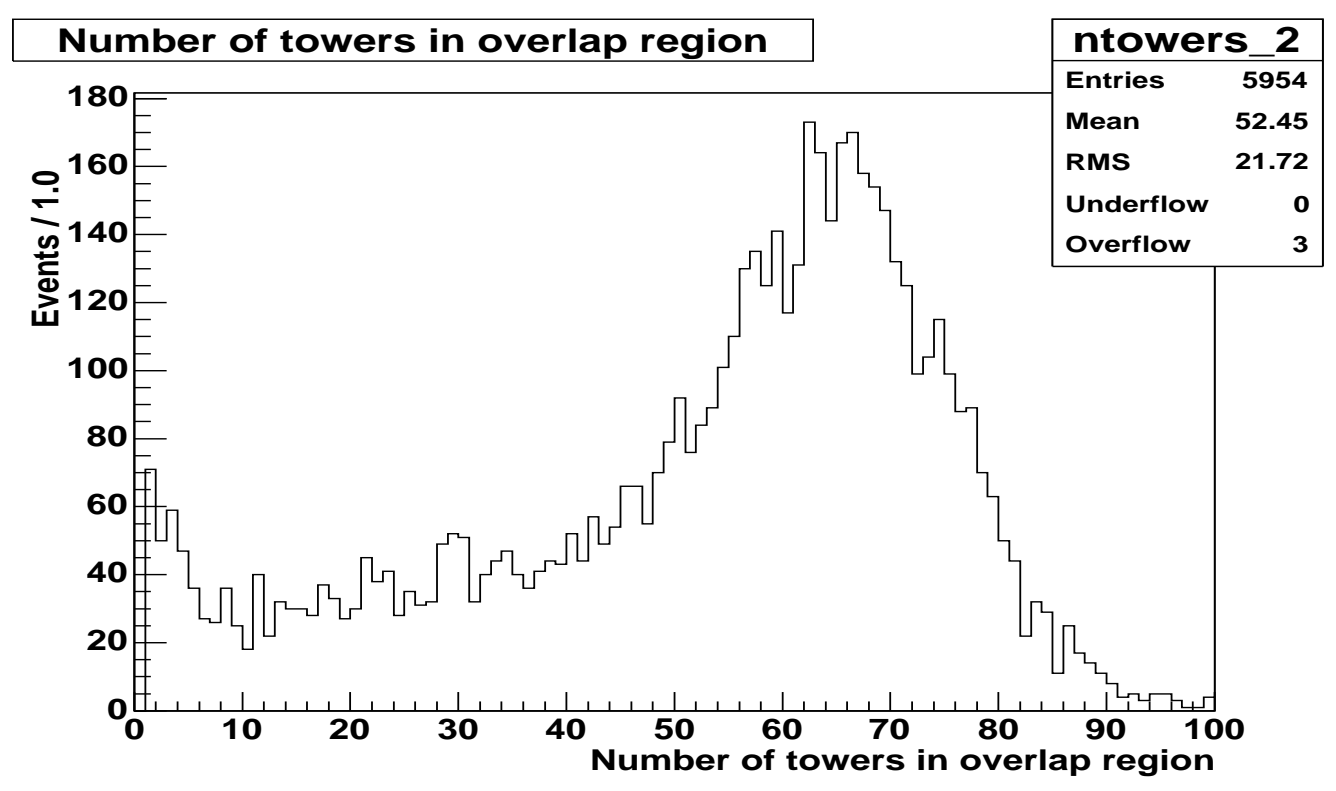

Figure 17: Number of towers overlapped for $T^{\prime}$ mass $1.75 \mathrm{TeV}$ 


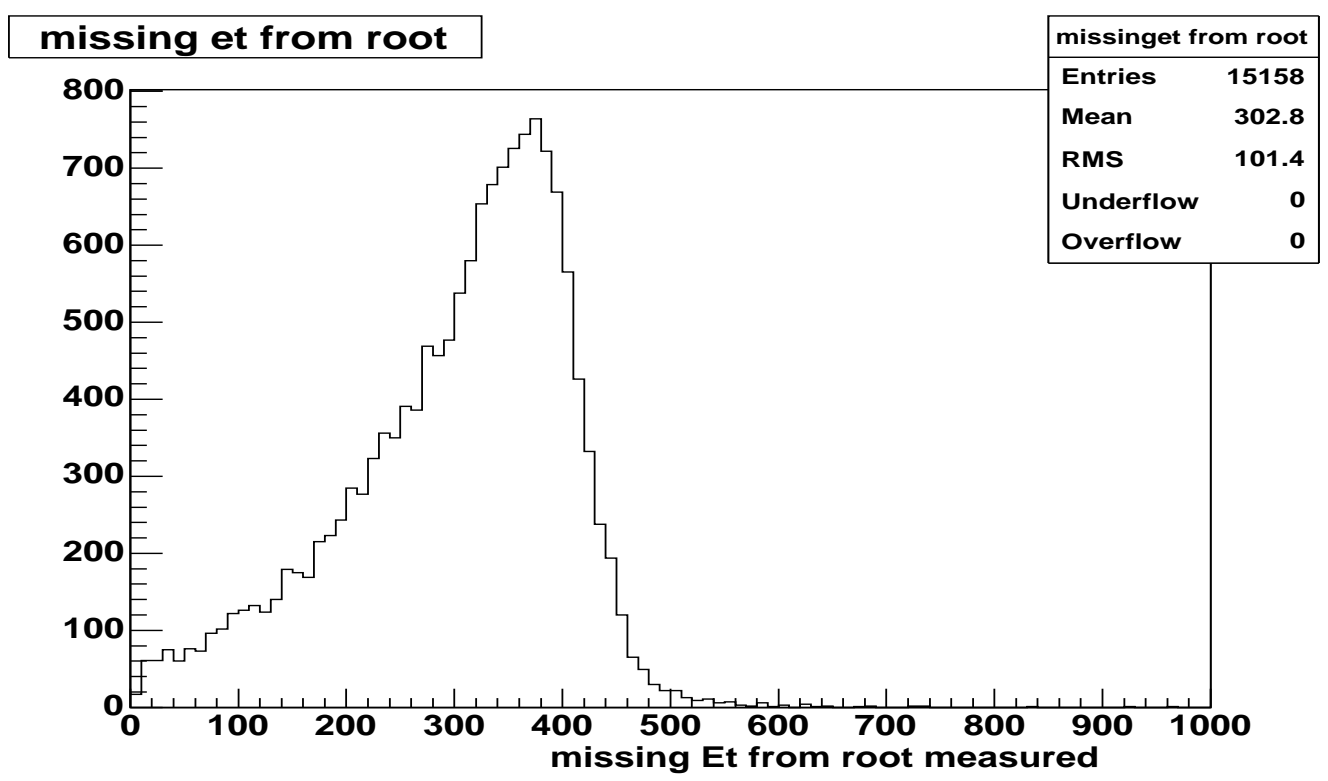

Figure 18: Missing $E_{t}$ for $1 \mathrm{TeV} T^{\prime}$ mass

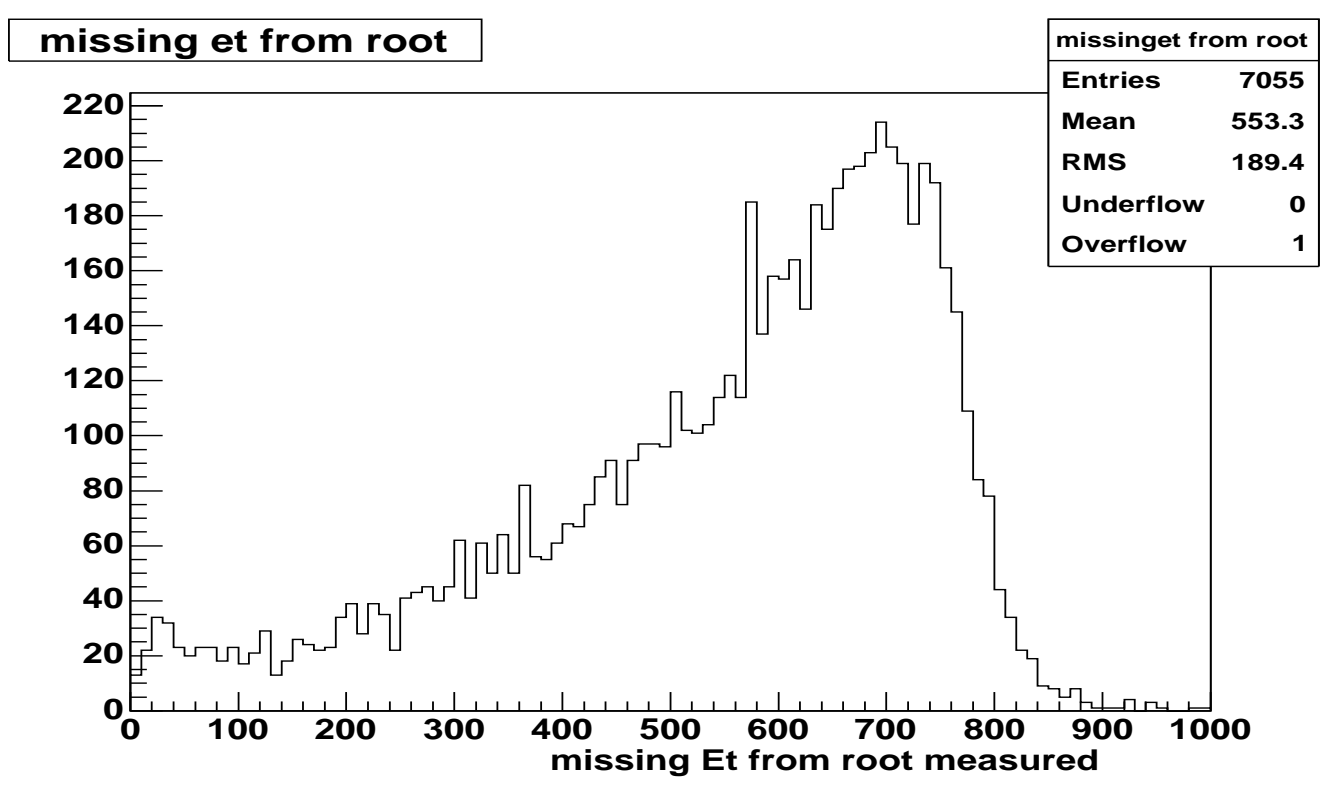

Figure 19: Missing $E_{t}$ for $1.75 \mathrm{TeV} T^{\prime}$ mass 


\section{Reconstruction of the t quark Using a New Jet Algorithm}

In this study, a new "cluster-mass" algorithm was used to reconstruct the t quark from the three jets. The algorithm is described in detail in reference [1] for the two-jet case. The main steps are summarized below, including how the algorithm was extended to the three-jet case:

-Find ten highest $E_{T}$ EM crystals in ECAL opposite the Z-boson to serve as a set of seed candidates (the ECAL has very fine segmentation and better energy resolution compared to the HCAL);

-From this set, identify the three crystals with the largest $E_{T}$ in the vector sum of three crystals, with at least 1.5 crystal separation between any two. These three crystals are the seeds of the energy clusters. For now, their $(\eta, \phi)$ are assumed to be the initial direction of the quarks;

-Next, the set of all CMS calorimeter towers in either cone around the seed directions was constructed. The cone used had a radius of 0.5 in $\eta-\phi$ space. Clearly, these three cone are overlap significantly as shown in Figures 20 and 21. Any tower not in any overlap region was assigned to the appropriate cluster;

-After ordering the towers in the overlap region by $E_{T}$, and starting with the most energetic one, each tower is assigned to one of the clusters depending on whether it increased the linear sum of the masses of the three clusters by the smallest amount. After all towers have been assigned, there exists three distinct energy clusters;

- To get a good estimate of the angle of the quark (in the absence of tracking), the energy-weighted centroid of the EM crystals in each cluster was used.

For the two-jet case, the overlap region is simply defined, and the assignment of towers in the overlap region to either of the two clusters follows unambiguously. However, for the three-jet case, the overlap region can be divided into four parts at most (see Figure 20 and Figure 21): one region where all three overlap, and three regions where only two overlap. For the three-jet case, a tower in any overlap region can be assigned to a cluster only if that cluster overlaps with another cluster in that region. That is, in the three regions where only two overlap, there are only two choices, while in the remaining region, there are three choices. Of course, sometimes only two cones overlap, and the assignment follows unambiguously. The algorithm is otherwise basically the same as the two-jet case. The only difference is that the topology of the three-jet case is more complicated than the two-jet cases.

Figures 22 and 23 show the distance $\Delta R$ in $\eta$ - $\phi$ space between the initial quark direction and the direction determined from each energy cluster. The peak difference is about 0.01 , which is about the tower size of the ECAL crystal. This is some assurance that the "cluster-mass" algorithm finds the correct quark direction. 


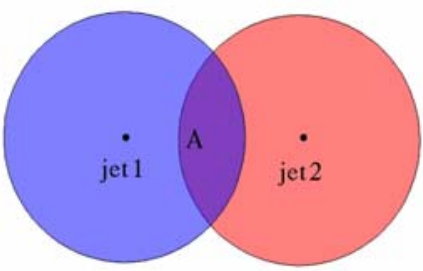

Figure 20: 2 jets overlapped

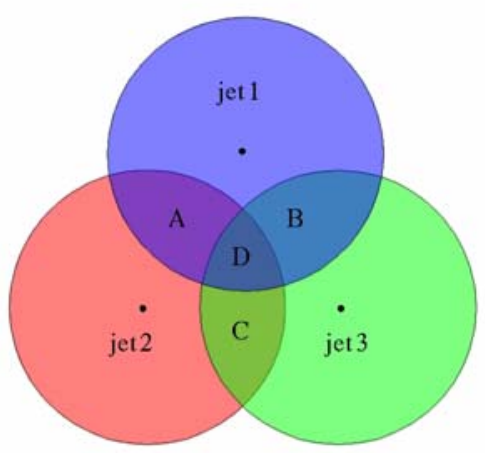

Figure 21: 3 jets overlapped 


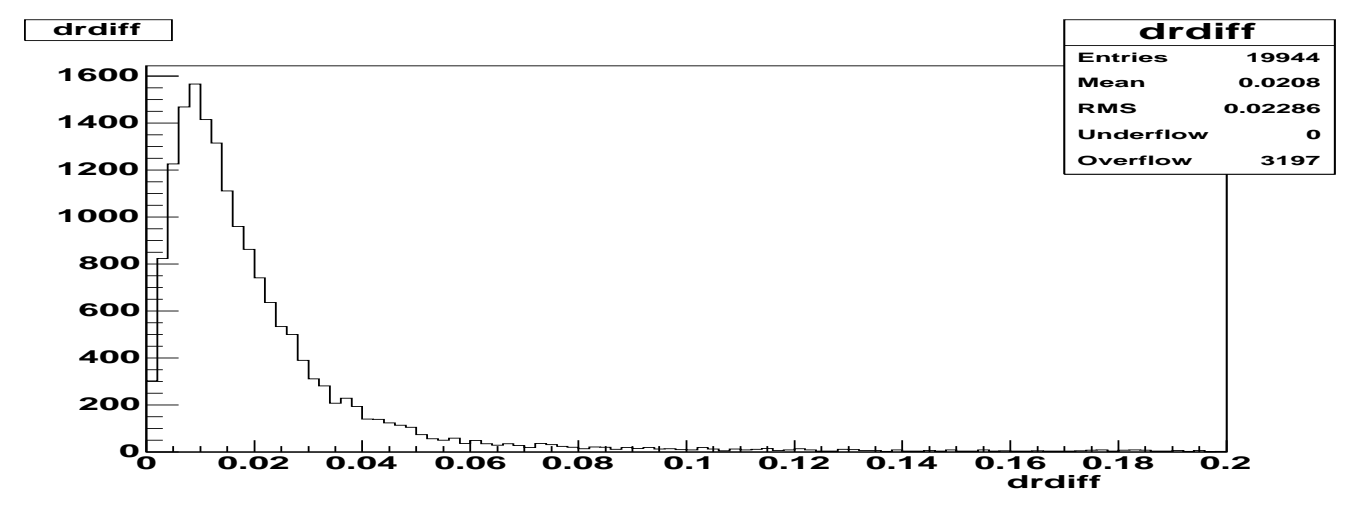

Figure 22: $\Delta R$ between quack direction and jet for $1 \mathrm{TeV} T^{\prime}$ mass

All this algorithm does is find three separate clusters to help narrow the window around the $\mathrm{t}$ quark mass. To separate the $t$ quark from the background further, there are two more possibilities to try:

- Identify the b quark;

- Reconstruct two clusters as the $\mathrm{W}$ boson;

For b quarks with such a large $P_{T}$, the conventional b-tagging algorithms are not efficient without inducing a large mis-tagging probability. Probably, a new, specialized algorithm is needed. The development of such an algorithm is beyond the scope of this note and expertise of the authors. The second possibility is to use the kinematics of the top decay, specifically the decay of the $\mathrm{W}$ boson. The next section will discuss this in detail.

\section{Reducing the Background Events from Standard Model}

The decay channel of the $t$ quark being studied has a three-body final state. After trying many different approaches, cutting on a Dalitz plot appeared to be the easiest and most straight-forward way to help reduce the backgrounds, and narrow the window around the reconstructed t quark mass further. A Dalitz plot is a way to represent the entire phase space, viz. all essential kinetically variables of any three-body final state in a scatter plot (first introduced by Dalitz in 1953).

In this case, $t \rightarrow W+b, W \rightarrow q+q$, the three-body final state consists of a $\mathrm{b}$ jet directly from the $\mathrm{t}$ quark decay, and two jets from the $\mathrm{W}$ decay. The main background to the T' signal comes from Standard Model production of $\mathrm{Z}$ plus multi-jets where the $\mathrm{Z}$ is nearly identical to the $\mathrm{Z}$ in the Little Higgs Model, $T \rightarrow Z+t$. However, for background, there is no correlation between the multi-jets in the $\mathrm{Z}$ plus multi-jets events. For these events, the physical region in a Dalitz plot is populated over a large allowed area, and in the absence of resonances or interferences, is nearly uniformly populated. In a Dalitz plot, resonant behavior of two of the final state particles gives rise to a bands of higher density, parallel to one or both of the coordinate axes, or along a 45 degree line.

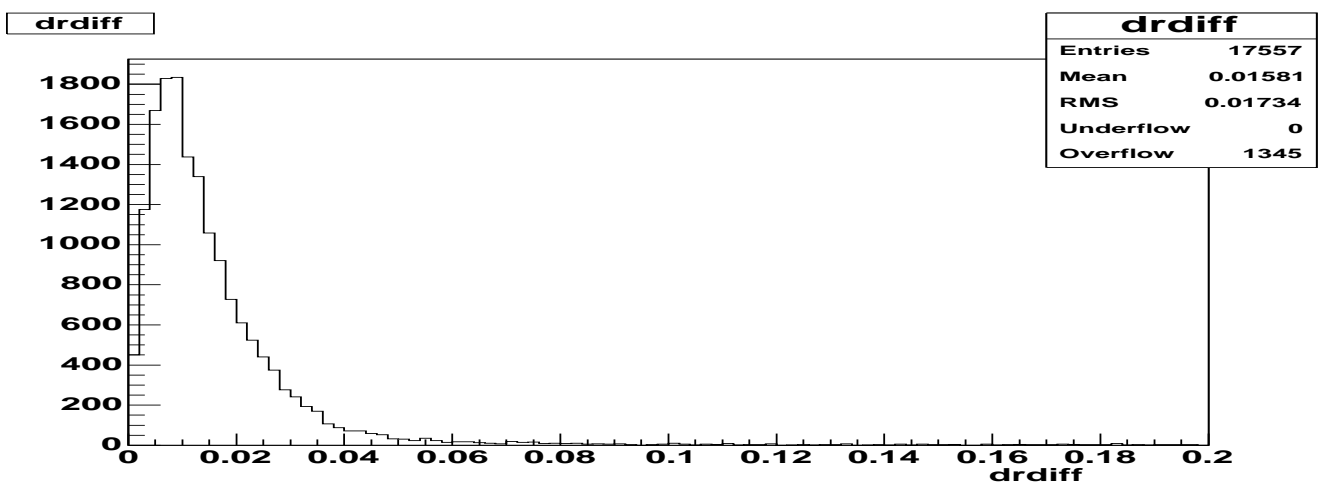

Figure 23: $\Delta R$ between quack direction and jet for $1.75 \mathrm{TeV} T^{\prime}$ mass 


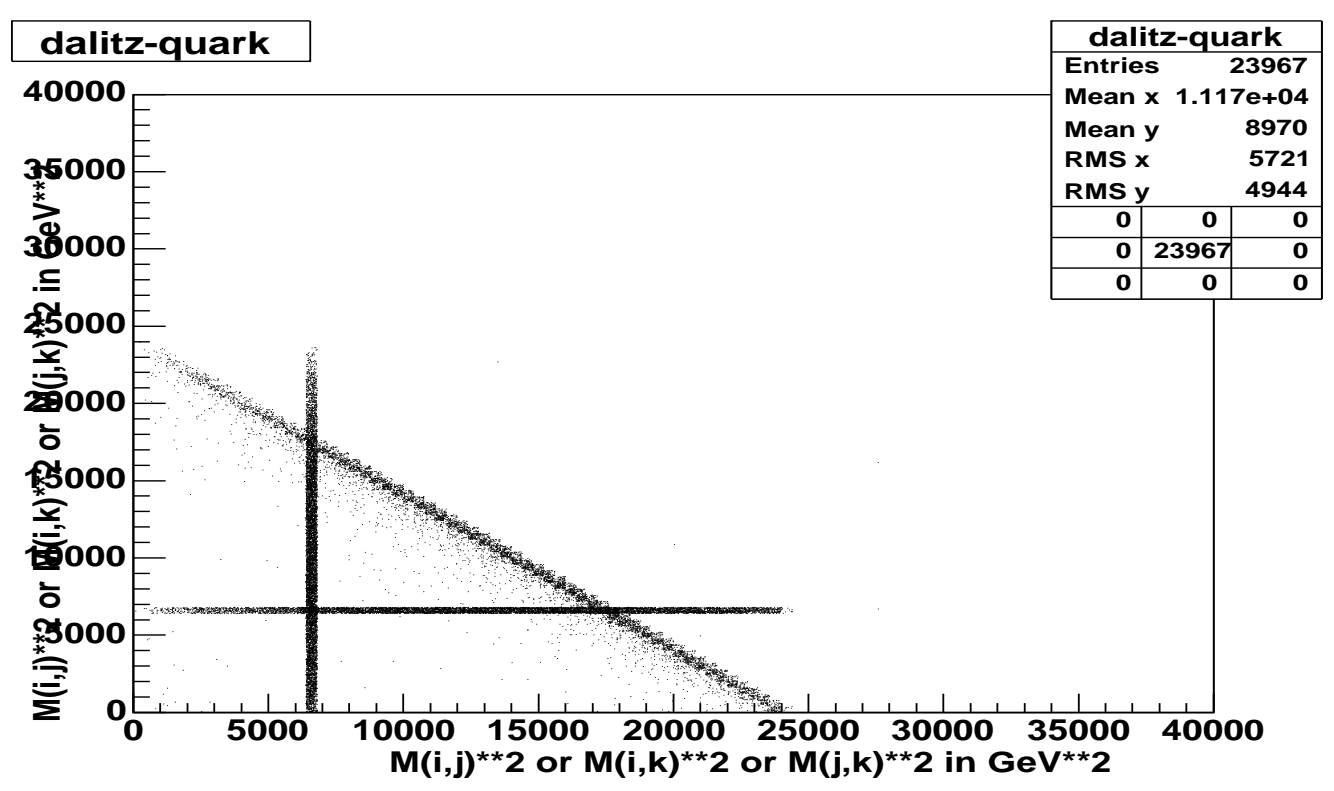

Figure 24: Dalitz plot using quarks for a $1.0 \mathrm{TeV} T^{\prime}$ mass in the Little Higgs Model

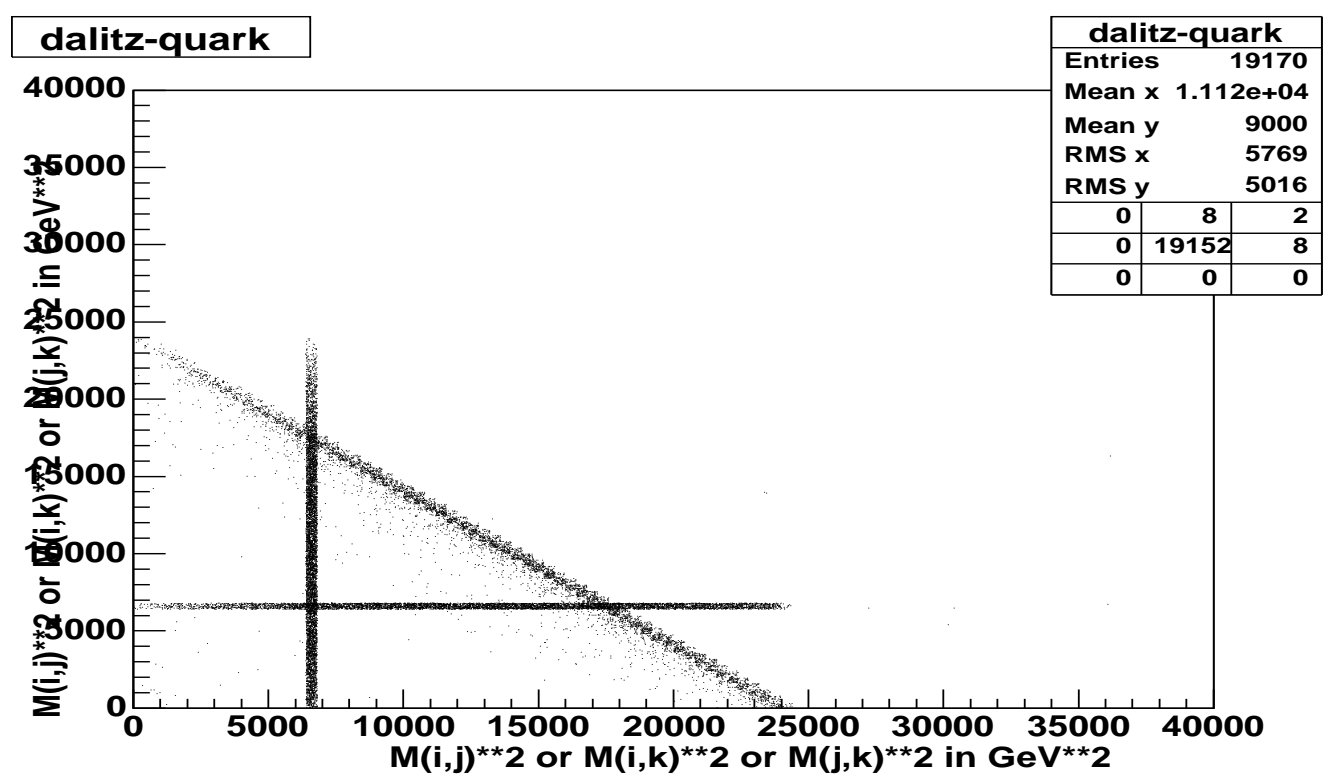

Figure 25: Dalitz plot using quarks for a $1.75 \mathrm{TeV} T^{\prime}$ mass in the Little Higgs Model

Figure 24 and 25 show the Dalitz plot using generator level quark information for T' masses of $1.0 \mathrm{TeV}$ and $1.75 \mathrm{TeV}$. The horizontal and vertical bands represent the two body decays of the $\mathrm{W}$ boson, The 45 degree band represents the two body combination in which the common assignment (particle 2) is the b quark, and the other two particles come from the $\mathrm{W}$ decay. 
The cuts we used is that the $M^{2}$ (i,j,k combination )> $1000 G e V^{2}$, and $M^{2}(\mathrm{i}, \mathrm{j}, \mathrm{k}$ combination) within the triangle of band of $30000 \mathrm{GeV}^{2}$, which is determined by band line plus about $20 \%$ resolution. The $\mathrm{M}$ means the di-jet mass of any possible combination of two body. Due to the calorimeter resolution and the mis-identification of jets(b jet or W jets), so the plot of Figure 26, is not like in Figure 24, as three lines. However, the density is much higher in the triangle region(Figure 26) than other area for the signal file(Figure 27), while for the background, the density is uniform, the cut will reduce $\mathrm{Z}$ plus multi-jets background very effectively, as well as to pick right jets to form the top, so improve the mass resolution. see the table 1.

Using Dalitz plot, which unlike $\mathrm{W}$ mass window or $\mathrm{t}$ quark mass window cuts, it is looking the resonant in the entire phase space. In order to see the effect by the Dalitz cut only, we calculate the invariant mass of the three jets as one "fat" jet before and after Dalitz cut only. We can see the Dalitz plot cut alone, already gave roughly right t quark mass with reasonable resolution. We used two different sets of "three jets", one is from the standard jet finding using cone algorithm in root file, or "Interactive Cone Algorithm with cone size 0.5"(ICA05). Another is using our "cluster-mass" algorithm. The differences between these two jets sets is that the one "fat" jet invariant mass from our new algorithm will get roughly right reconstructed t quark, but the ICA05 jets set has much larger tail(Figure 28-29) and (Figure 30-31). 


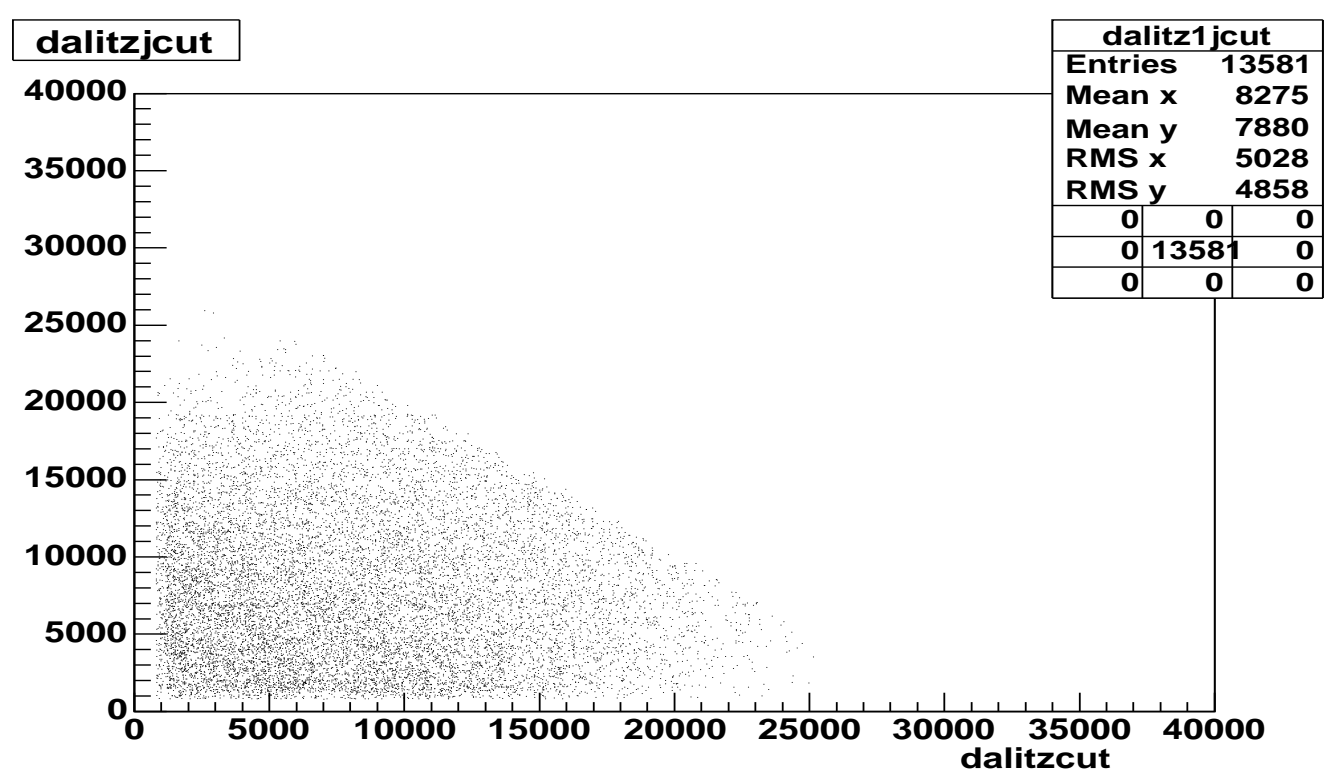

Figure 26: Dalitz plot inside of cuts using "cluster-mass" jet set for $1 \mathrm{TeV} T^{\prime}$ mass

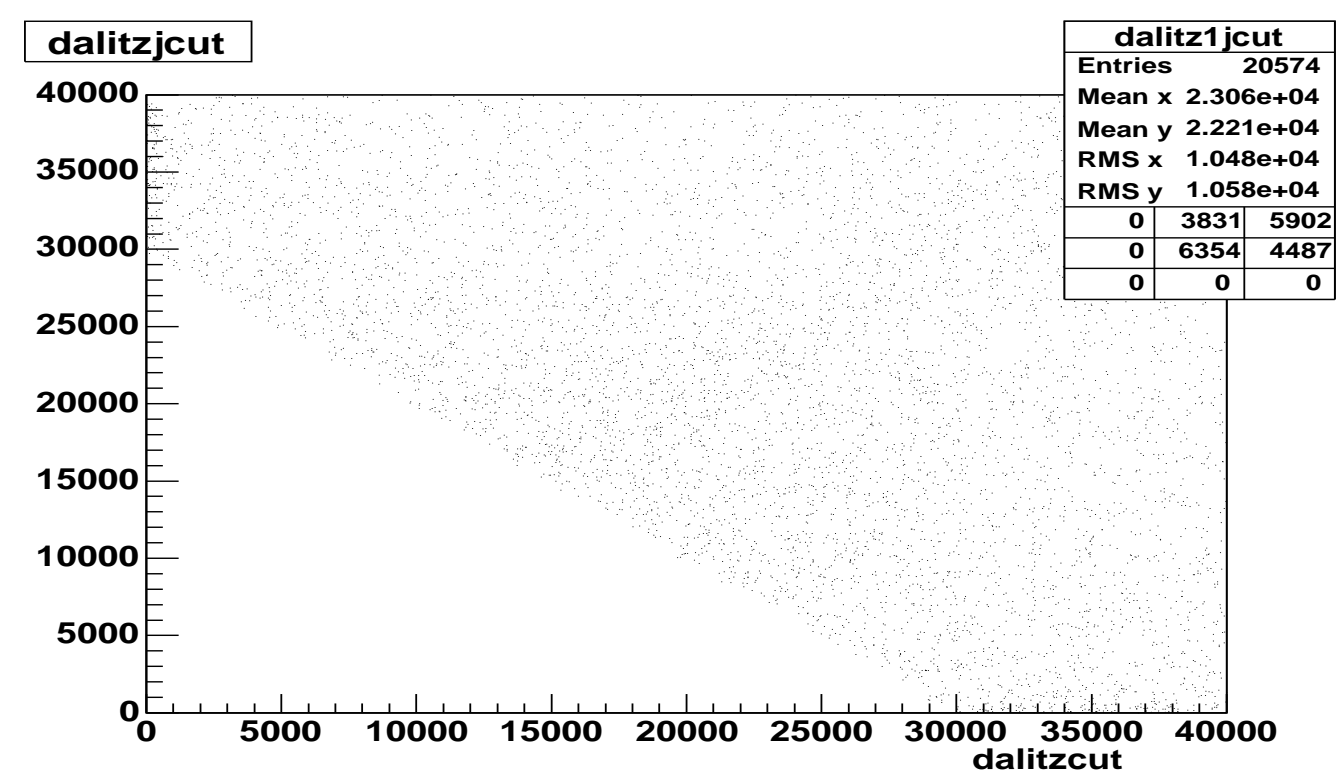

Figure 27: Dalitz plot outside of cuts using "cluster-mass" jet set jet set for $1 \mathrm{TeV} T^{\prime}$ mass 


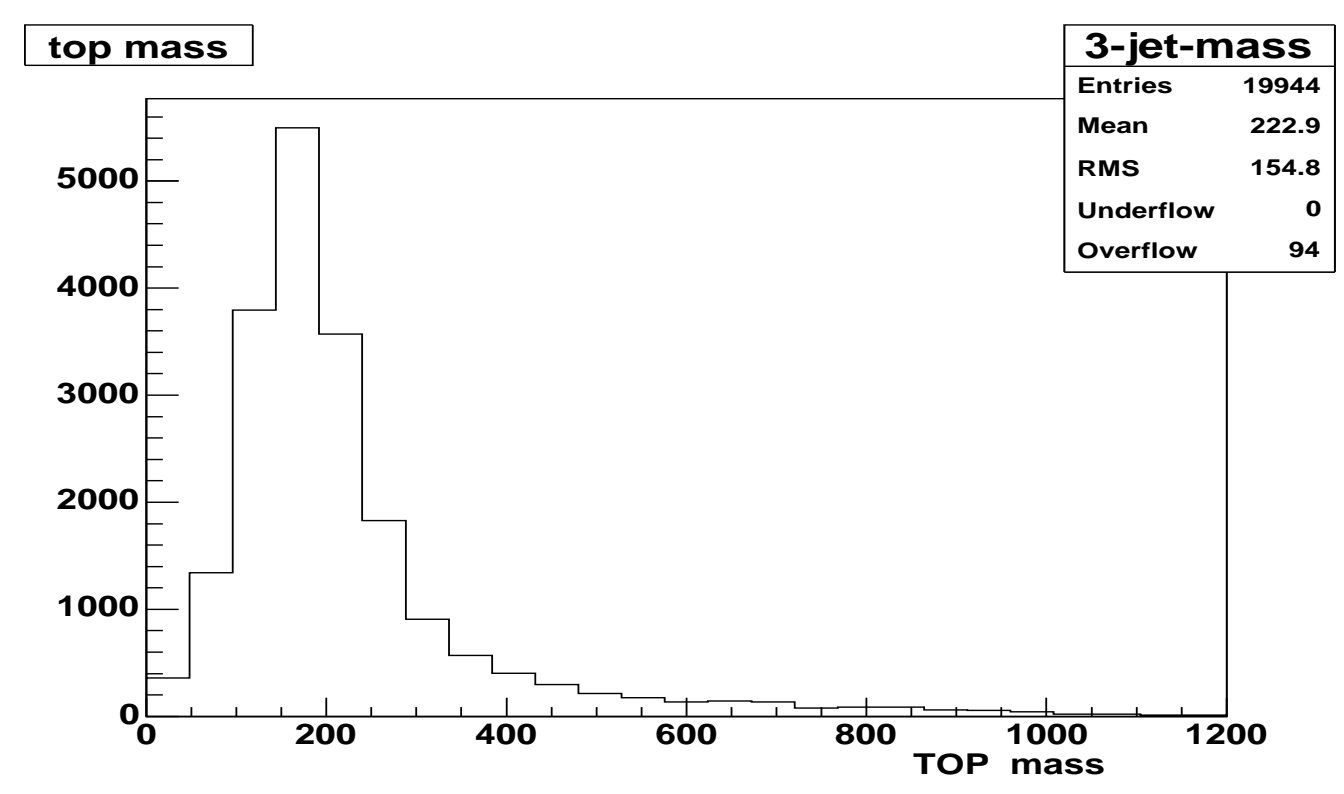

Figure 28: Three jet invariant mass using "cluster-mass" jet set for $1 T^{\prime}$ mass

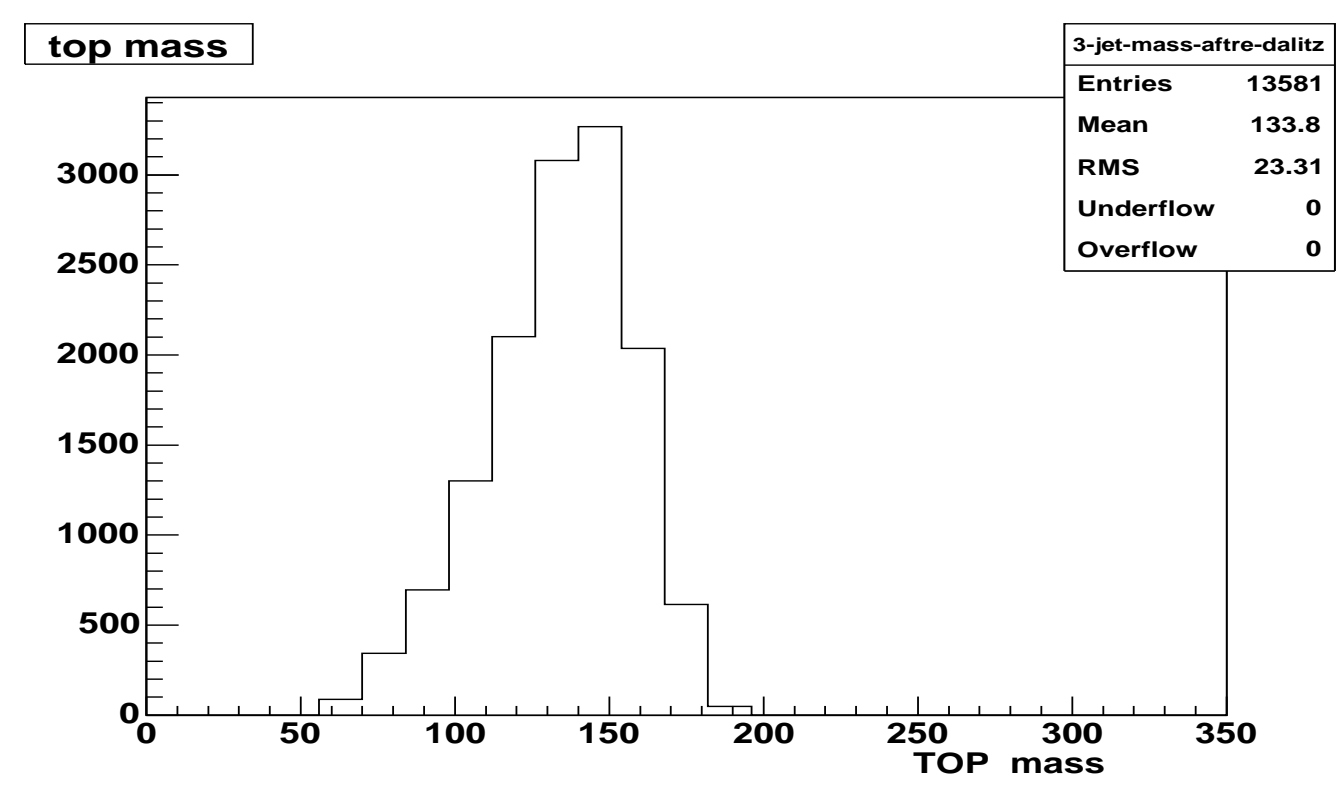

Figure 29: Three jet invariant mass after Dalitz cuts using "cluster-mass" jet set for $1 T^{\prime}$ mass

We can see that using "cluster-mass" jet set, it already picks right of three jets from the top quark most of time, with some wrong jets which causing the tail(Figure 28). After Dalitz cut, we get rid of the wrong jets, and get the right top peak(Figure 29). Since the jets is not calibrated, so it did not sit at $175 \mathrm{GeV}$. 


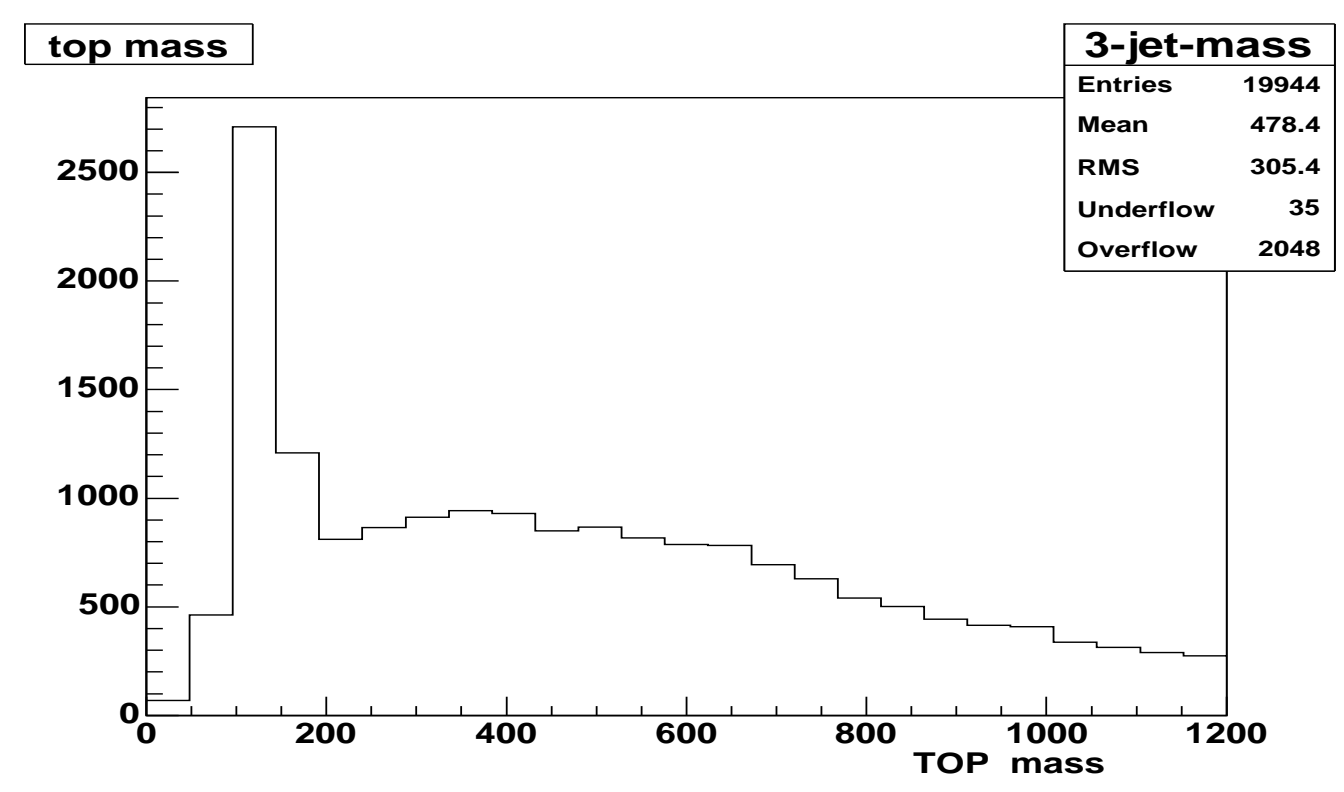

Figure 30: Three jet invariant mass using "ICA05" jet set for $1 \mathrm{TeV} T^{\prime}$ mass

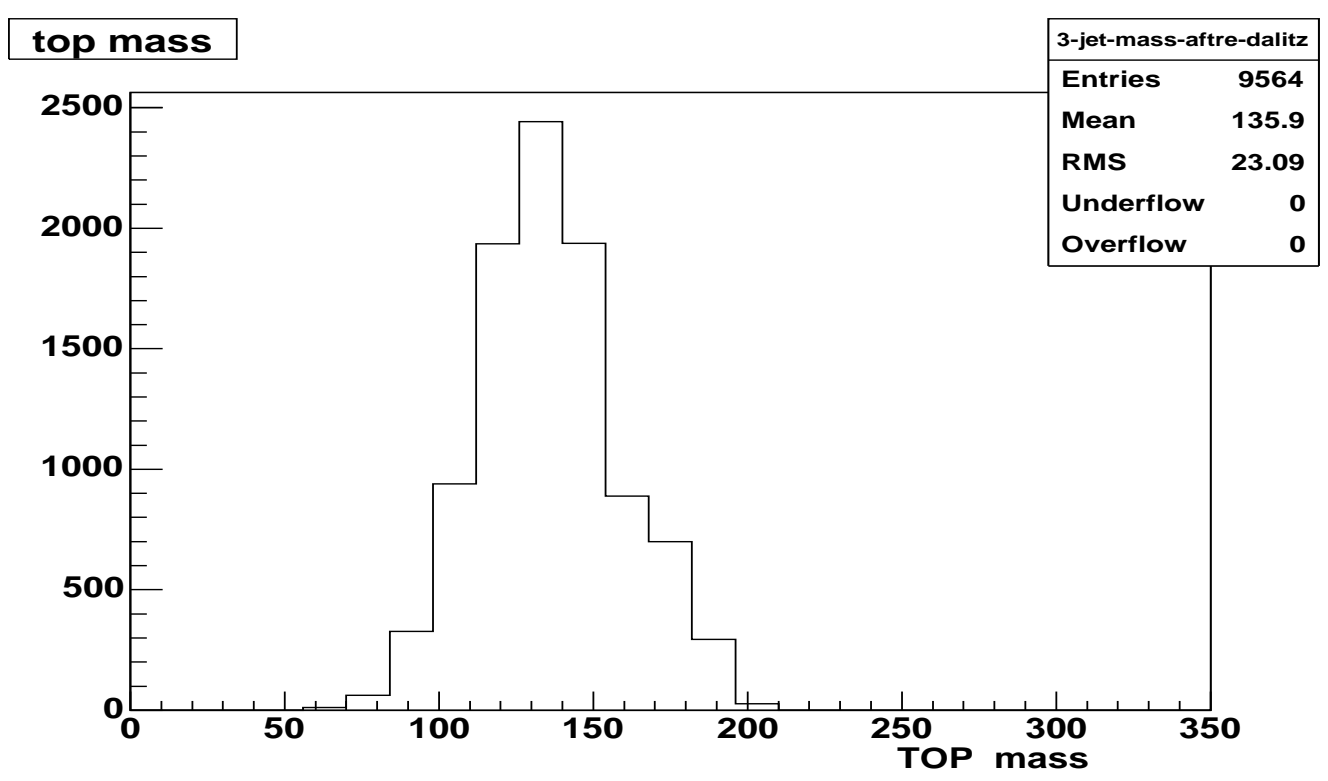

Figure 31: Three jet invariant mass after Dalitz cuts using "ICA05" jet set for $1 \mathrm{TeV} T^{\prime}$ mass

We can see that using "ICA05" jet set, it has much larger tail compared to the "cluster-mass" jet set(Figure 30), because the ICA05 jets set, have many overlapped jets, which over-counting the jets energy. However, After Dalitz cut, we get rid of the most of tail, because the Dalitz plot cut picks jets with right w and top mass region(Figure $31)$. 


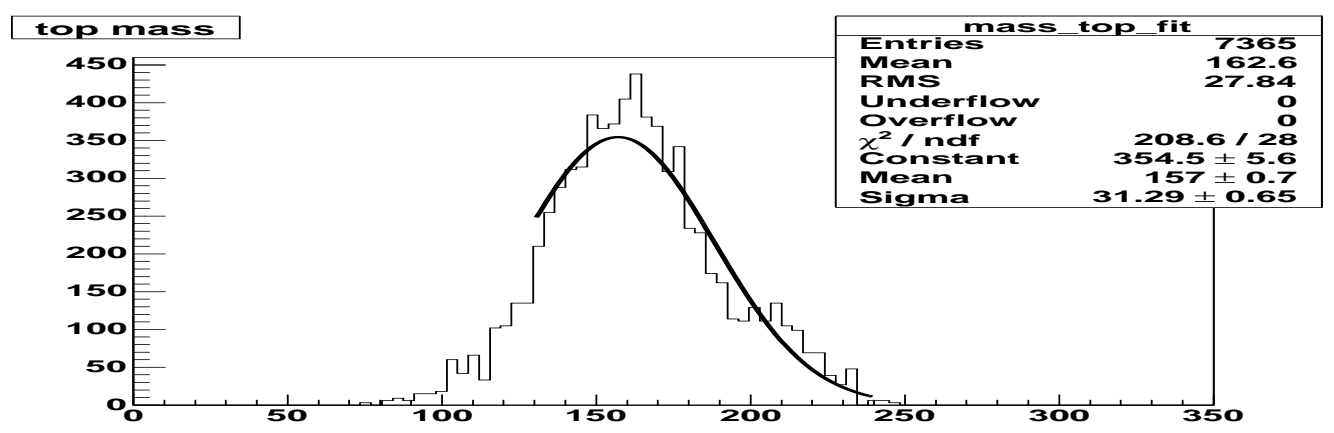

Figure 32: The t quark mass distribution using ICA05 jet set for $1 \mathrm{TeV} T^{\prime}$ mass

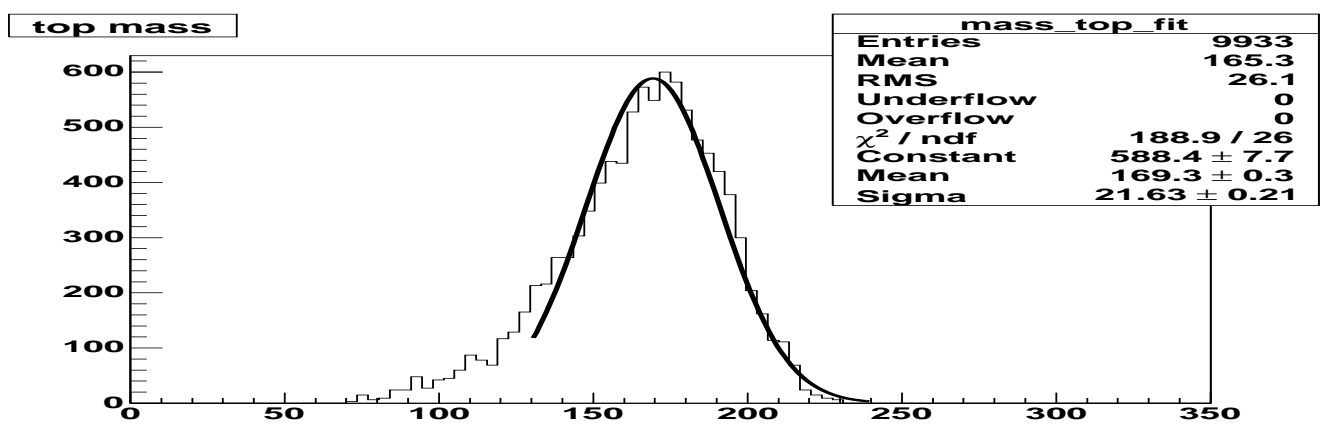

Figure 33: The t quark mass distribution using "cluster-mass" jet set for $1 \mathrm{TeV} T^{\prime}$ mass

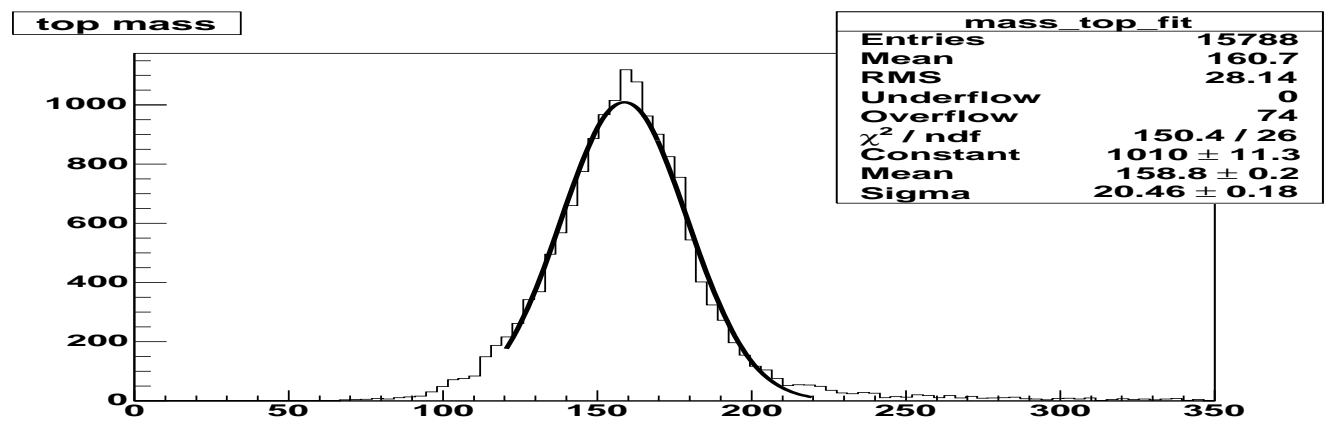

Figure 34: The t quark mass distribution using quark direction for $1 \mathrm{TeV} T^{\prime}$ mass 


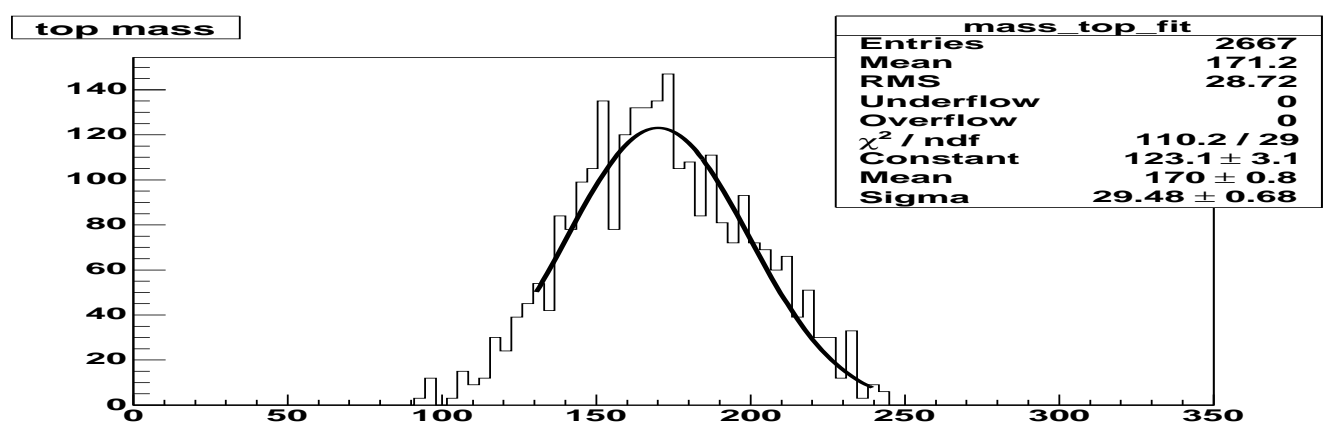

Figure 35: The t quark mass distribution using "ICA05" jet set for $1.75 \mathrm{TeV} T^{\prime}$ mass

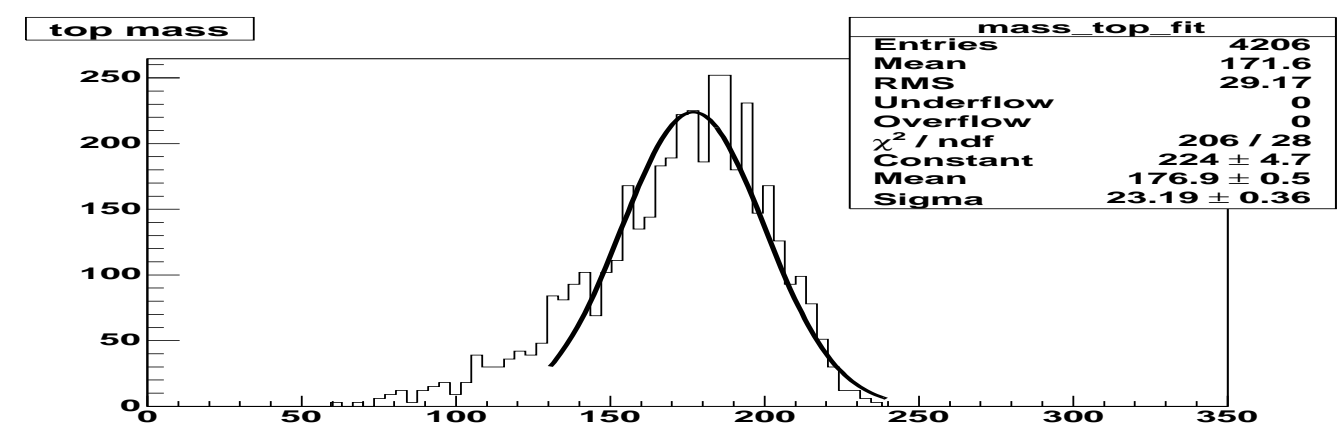

Figure 36: The t quark mass distribution using "cluster-mass" jet set for $1.75 \mathrm{TeV} T^{\prime}$ mass

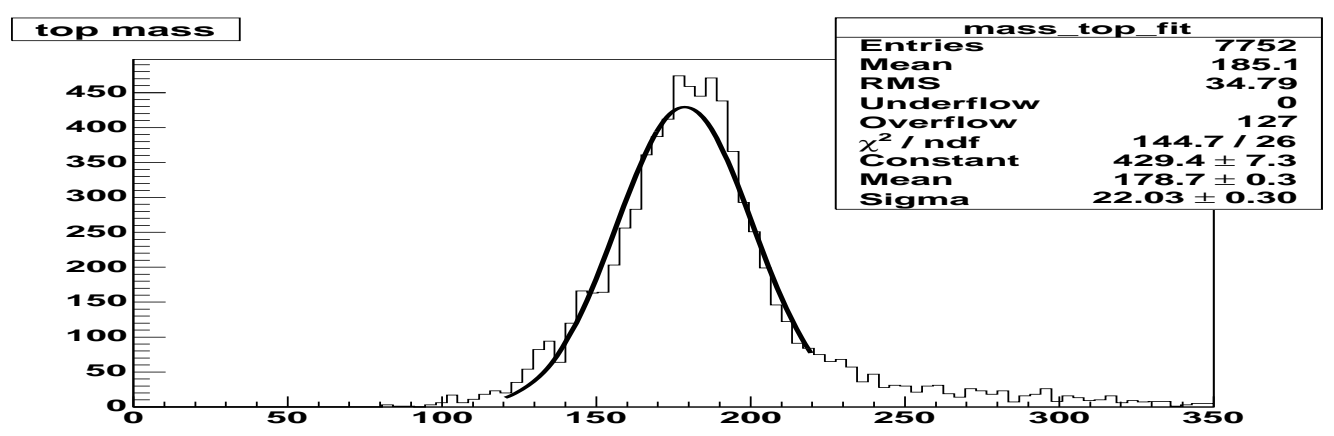

Figure 37: The t quark mass distribution using quack direction for $1.75 \mathrm{TeV} T^{\prime}$ mass 
Table 1: The top mass resolution with different algorithms for $1 \mathrm{TeV}$ and $1.75 \mathrm{TeV} T^{\prime}$ mass

\begin{tabular}{lcc}
\hline \hline & $1 . \mathrm{TeV}$ & $1.75 \mathrm{TeV}$ \\
\hline ICA05 & $19.9 \%$ & $17.4 \%$ \\
Cluster-Mass & $12.7 \%$ & $13.1 \%$ \\
Quark direction & $12.7 \%$ & $12.3 \%$ \\
\hline
\end{tabular}

From Figure 32 to Figure 37, We can see that the t quack mass resolution to use the "quack" direction is the best. If we use standard ICA05 jets set, the resolution could be $50 \%$ worse. However, by using the "cluster-mass" algorithm, the resolution is nearly as good as that obtained using "quack" direction, which means we have minimized the "angle"(jet direction) error. Top mass resolutions are quoted in Table 1. Since the top mass resolution plays important role in the $T^{\prime}$ reconstructed mass resolution, therefore the ratio of the signal to the background, it is essential to use "cluster-mass" algorithm in this study which High $P_{t}$ jets are involved.

\section{Analysis and Results}

The generation, simulation and reconstruction of $Z$ plus multi-jets background is described in Ref[1], there are preselection cuts on $P_{T}$ of $\mathrm{Z}$ boson, to make data production more sufficient. So the first cut we did for our analysis was to make cuts on $P_{T}$ of $\mathrm{Z}$ boson to require $300 \mathrm{GeV}$ above. The cross section of $\mathrm{Z}$ plus multi-jets background is factor of a few hundred larger than the signal $(1 \mathrm{TeV})$, as seen in Table 2, therefore we have to find some strategy to reduce the background while not significantly reducing the signal.

Table 2: Z+NJets cross-sections times branching ratios, $\sigma(f b) \times B R$ (where $P_{T}^{Z}>300$. GeV)

\begin{tabular}{lcccc}
\hline \hline Sample & $\mathrm{Z}+1$ jet & $\mathrm{Z}+2$ jets & $\mathrm{Z}+3$ jets & $\mathrm{Z}+4$ Jets \\
\hline$Z \rightarrow e^{-} e^{+}$ & 487 & 1027 & 1405 & 1245 \\
$Z \rightarrow \nu \bar{\nu}$ & 2922 & 6162 & 8430 & 7470 \\
\hline
\end{tabular}

As we have mentioned above, there is a forward jet in the "Little Higgs Model" sample produced by W fusion. Figure 38 and 39 show the scalar plot of $\eta$ vs $E_{t}$ of the first four leading jets. We can see that the signal sample(Figure 38) has many jets distributed beyond the $\eta 3$, and very few jets $E_{t}$ is larger than $400 \mathrm{GeV}$, while the sample of $\mathrm{Z}$ plus multi-jets(plot show the $\mathrm{Z}$ plus three jets, Figure 39), almost no jet activity beyond $\eta 3$, and much more jets have Et larger than $400 \mathrm{GeV}$. Hence, we apply the forward jet tagging at "cut C". 


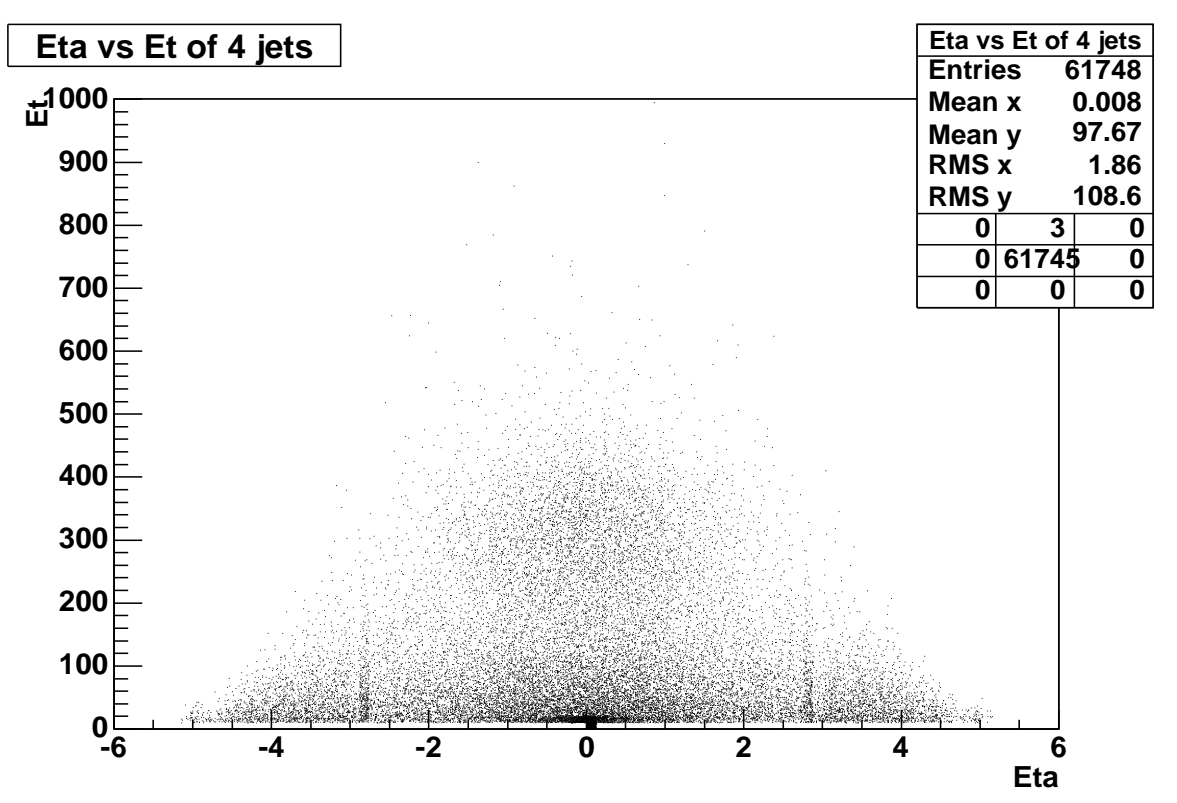

Figure 38: $\eta$ vs $E_{t}$ of jets for signal sample

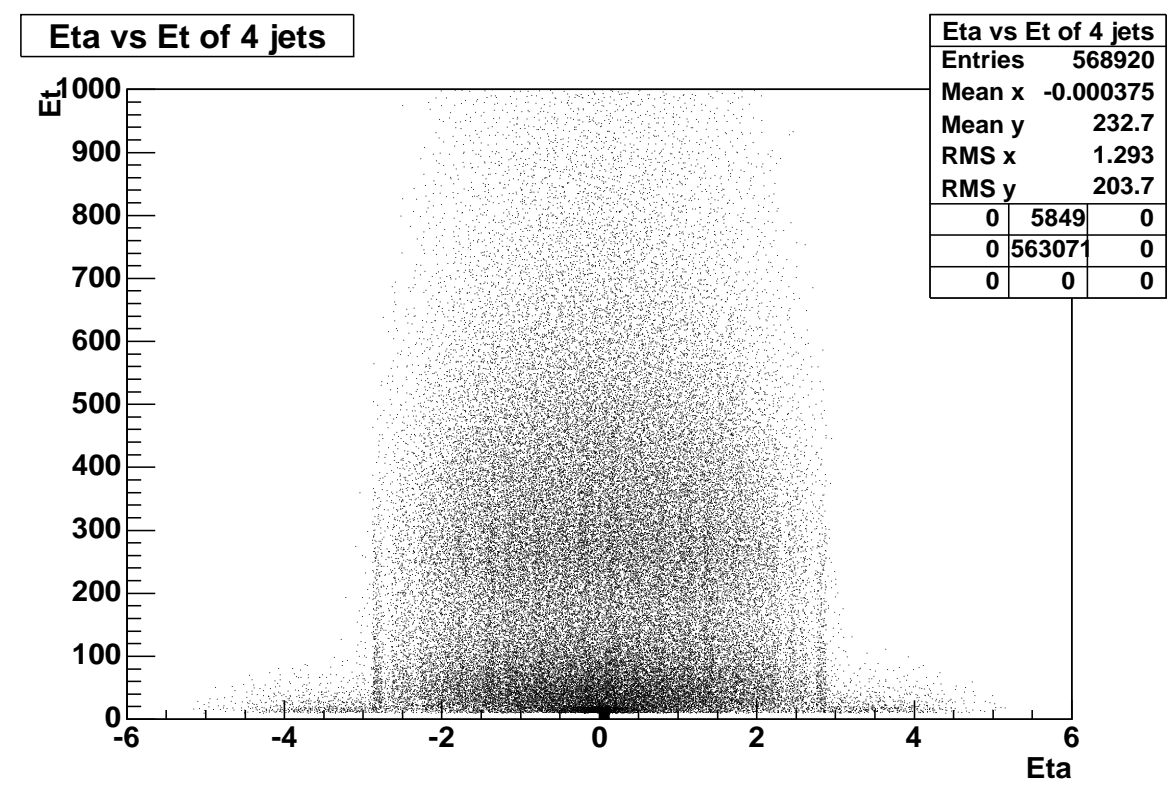

Figure 39: $\eta$ vs $E_{t}$ of jets for $\mathrm{Z}$ plus 3 jets sample 
Table 3: Analysis result

\begin{tabular}{lccccccc}
\hline \hline Sample & $\sigma \times \mathrm{Br}(\mathrm{fb})$ & No of events & cut a & cut b & cut c & cut d & cut e \\
\hline $1 \mathrm{TeV} T^{\prime}$ & 25.6 & 19944 & 9933 & 9837 & 5367 & 4707 & 4440 \\
\hline $\mathrm{Z}+1$ jet & 2922 & 50389 & 14160 & 8790 & 1527 & 1143 & 771 \\
\hline Z+2jets & 6162 & 102481 & 22152 & 13803 & 1233 & 975 & 528 \\
\hline Z+3jets & 8430 & 143472 & 17655 & 13986 & 804 & 651 & 219 \\
\hline Z+4jets & 7470 & 124977 & 5277 & 3741 & 84 & 69 & 24 \\
\hline
\end{tabular}

Note:

cut a: $P_{T}>300 \mathrm{GeV}$, Dalitz cut and require at least three hits in ECAL;

cut b: $Z_{T}>100 \mathrm{GeV}$, and leading jet $P_{T}>50 \mathrm{GeV}$;

cut c: forward jet tagging, $E_{T}>20 \mathrm{GeV}$ and $|\eta|>3$;

cut d: angle cut in top rest frame;

cut e : all jet $E_{T}<400 \mathrm{GeV}$;

Another important background is QCD multi-jets plus $\mathbb{E}_{T}$. Esen and Harris have provided such data sample as root file with many different jet $P_{T}$ region, from very low $P_{T}$ to very high $P_{T}$, the cross section varies from $10^{6}$ $\mathrm{pb}$ to $0.01 \mathrm{pb}$, we have made analysis through all data samples(few thousands each), not a single event left after all analysis cuts. Clearly, we may don't have enough data sample to exam due to huge cross section of these QCD jets, specially for low $P_{T}$ data sample, however, we will focus on the $\mathrm{Z}$ plus multi-jets background only in this analysis, and expect that by our such tough analysis cuts, the QCD plus multi-jet background is not critical for this analysis.

The analysis cuts are listed in Table 3 . Here we just describe the cut $d$, which is the maximum angle between jets in the top quark rest frame(Figure 40,41). The top quark, decays into b quark and the $\mathrm{W}$ boson, so in the rest frame of the top quark, the $\mathrm{W}$ and $\mathrm{b}$ should be back to back, the jets from the $\mathrm{W}$ decay should be more or less also in the opposite direction of the $\mathrm{b}$ jet because the high $P_{T}$. So the angle between jets, take the maximum one, likely is the angle between the $b$ jet and the one of quark jets from the $\mathrm{W}$ decay, so it peaked at the 180 degree for "Little Higgs Model" data sample, while for Z plus multi-jets background, has no such behavior in the "three jets system rest frame" (equivalent to the top quark rest frame), which means that there is no such angle cooration. However, since we have to do the Dalitz plot cut first, the Dalitz plot used same information with this angle, so the benefit is not as big as we expected after Dalitz cut. However, it is still useful, so we kept this cut for our analysis procedure.

Once we have reconstructed t quark, we can calculate the transverse mass of the $T^{\prime}$ using the $E_{T}$ information. We plot the transverse mass of the $\mathrm{Z}$ and top quark(or three jets) system, normalized to an integrated luminosity of $\left(\sim 300 \mathrm{fb}^{-1}\right)$. The significance, defined as $\mathrm{S} / \sqrt{b}$ is 10.4 . Since the $E_{T}$ is not well measured and not well calibrated, as well as the jets energy, the result could be further improved(Figure 42). Our focus of this study is not on the $\mathbb{E}_{T}$ and jets calibration, rather, it is to focus on the reconstruction of the three jets(top) system. Since the "Little Higgs Model" is not the only theory to produce the $T^{\prime}$, other model may have much larger cross section. Our algorithm and analysis procedure is good for general purpose. We suppose the cross section is factor of 10 larger, we plot the same normalized plot for $\sim 300 \mathrm{fb}^{-1}$, we can see that the $T^{\prime}$ is well sit above the background(Figure 43). 


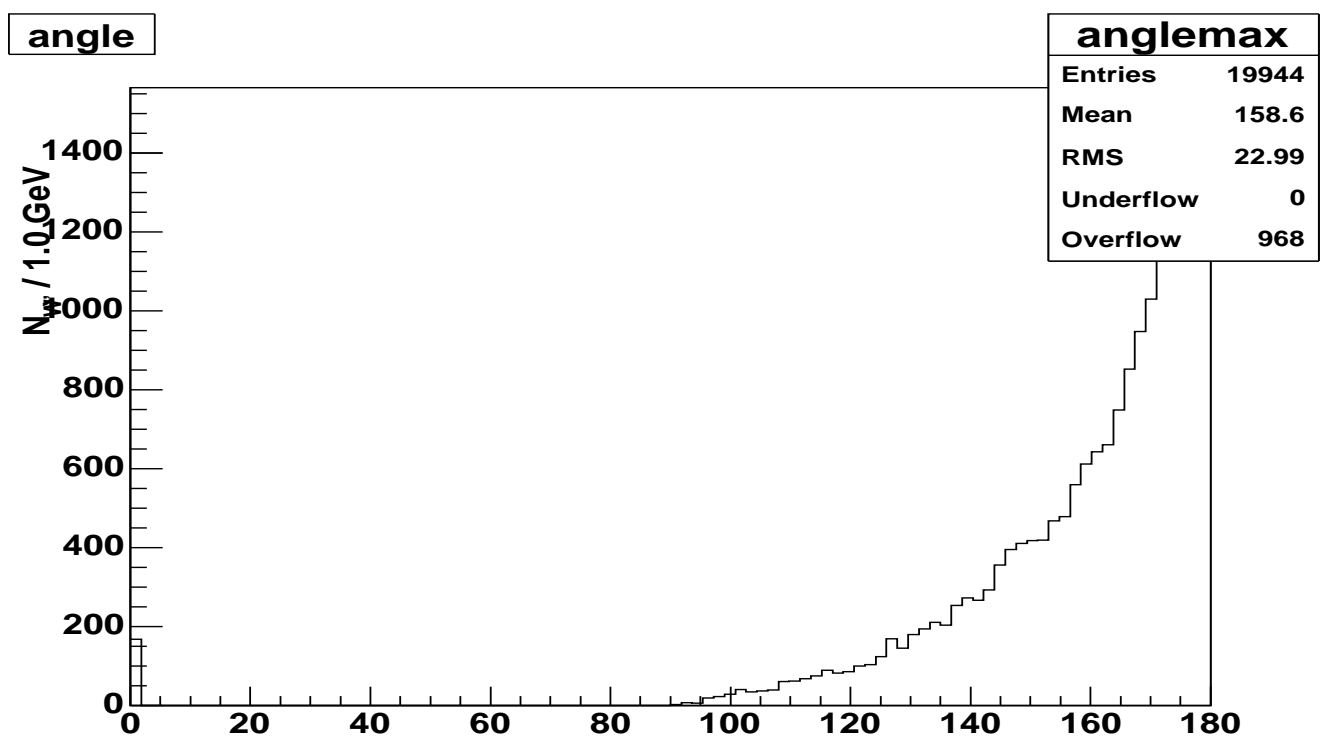

Figure 40: Maximum angle between jets in the three jets system rest frame for $1 \mathrm{TeV} T^{\prime}$ mass

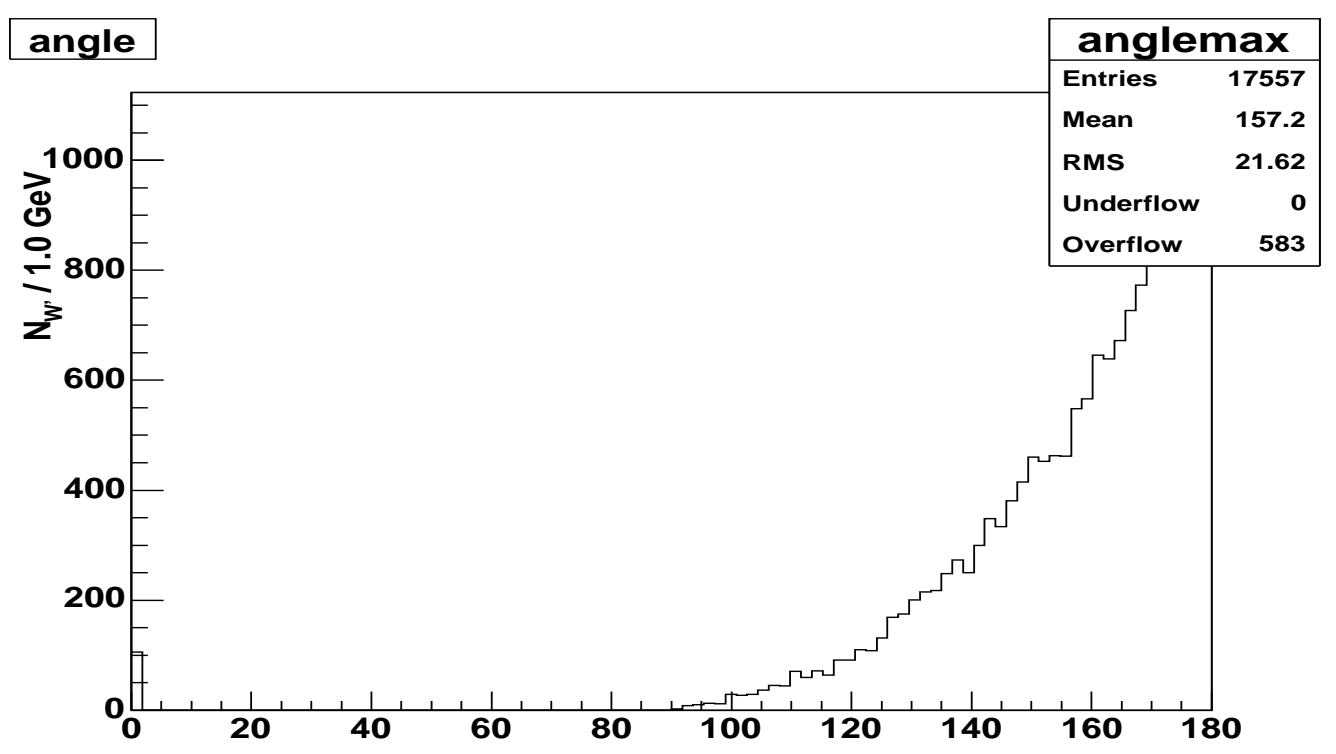

Figure 41: Maximum angle between jets in the three jets system rest frame for $1.75 \mathrm{TeV} T^{\prime}$ mass 


\section{Transverse Mass: $Z+T$}

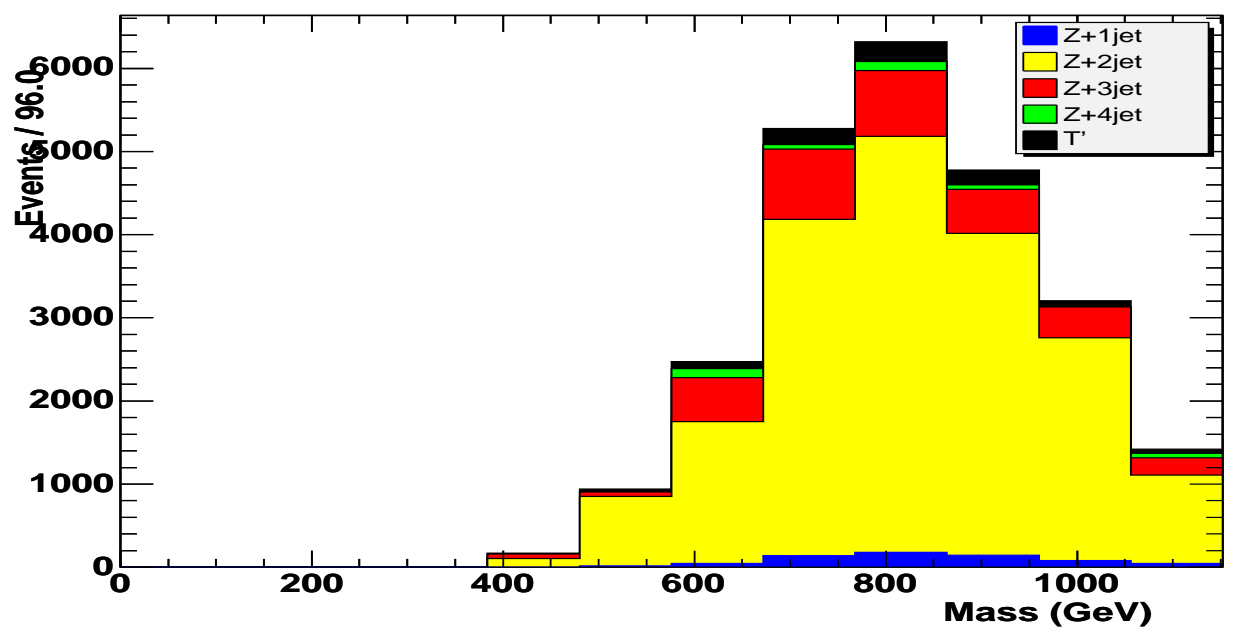

Figure 42: Normalized plot for $1 \mathrm{TeV} T^{\prime}$ transverse mass

Transverse Mass: $Z+T$

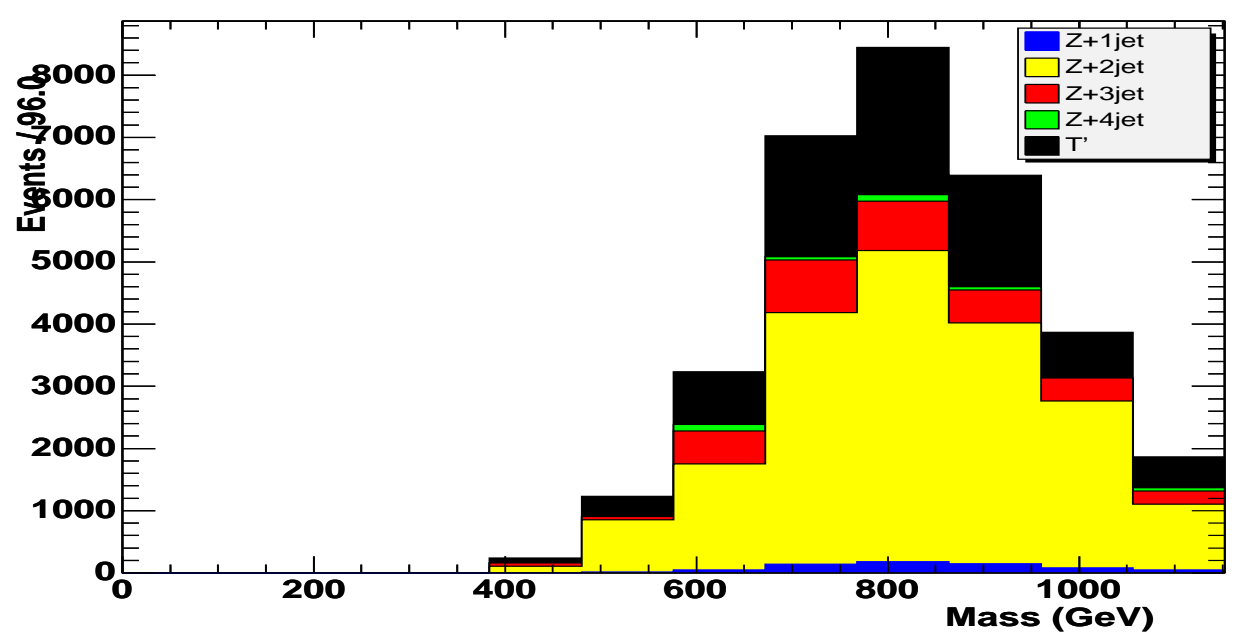

Figure 43: Normalized plot for $1 \mathrm{TeV} T^{\prime}$ transverse mass suppose the $T^{\prime}$ cross section is factor 10 larger 


\section{Summary and Conclusions}

This study demonstrated how to reconstruct the very boosted three jets system using our so called "cluster-mass" algorithm. We can reach a top quark(three jets system) mass resolution of about $12.7 \%$ which is limited by our calorimeter performance, and the algorithm almost eliminated deviation from the angle(jet direction) error. This channel is not the best choice to study "Little Higgs Model", or search the new particle, such as $T^{\prime}$, because the cross section is very small, the background is enormously. Specially, for $\mathrm{Z}$ decays into neutrinos channel, it requires very good $E_{T}$ measurement and calibration with environment of multi-high- $P_{T}$-jets appeared. However, we demonstrated we still can do it. We also show that the Dalitz plots cuts plays major role in the analysis procedure. There are some other model which deal with $\mathrm{T}^{\prime}$ decay, they may have much larger cross section compared to the Little Higgs Model. This study provided the algorithm to analyze high $P_{T}$ data samples in general.

\section{Acknowledgments}

We would like to express our thanks to Rick Vidal and Dan Green, for their very useful suggestions, ideas and great help, and to Stephen Mrenna, he helps us to establish the Pythia data card, so we were able to generate the "Little Higgs Model" signal with Pythia. We also wish to thanks to Robert Harris and Selda Esen, they provided the QCD jets plus $\mathscr{E}_{T}$ data samples for us as a background files with different jet Pt region. We give our special thanks to the CMS Fermilab physics group for useful comments and criticism during the course of this analysis and

preparation of this note. Also, many thanks to Hans Wenzel and CMS computing group for their strong support on the computer resources they provided.

\section{References}

[1] R. Vidal, Searching for Heavy New Particles Decaying into Di-Bosons at CMS CMS-IN Note 2006-004

[2] N. Arkani-Hamed, A. G. Cohen, T. Gregoire and J. G. Wacker, JHEP 0208, 020 (2002) [arXiv:hep$\mathrm{ph} / 0202089]$.

[3] N. Arkani-Hamed, A. G. Cohen, E. Katz, A. E. Nelson, T. Gregoire and J. G. Wacker, JHEP 0208, 021 (2002) [arXiv:hep-ph/0206020].

[4] Phenomenology of the Little Higgs Model

Phys.Rev.D67:095004,2003

Tao Han, Heather E. Logan, Bob McElrath and Lian-Tao Wang

University of Wisconsin

[5] Jet/MetAnalysis, HCAL/JET/MET Ntuple Maker, Pal Hidas

http://www.kfki.hu/ hidas/JetMetAnalysis/index.html 